\title{
NG STATE
}

\section{FTMS LABORATORY FOR HUMAN HEALTH RESEARCH}

\section{GlycoHunter}

\section{User's Manual for Release 1.00}

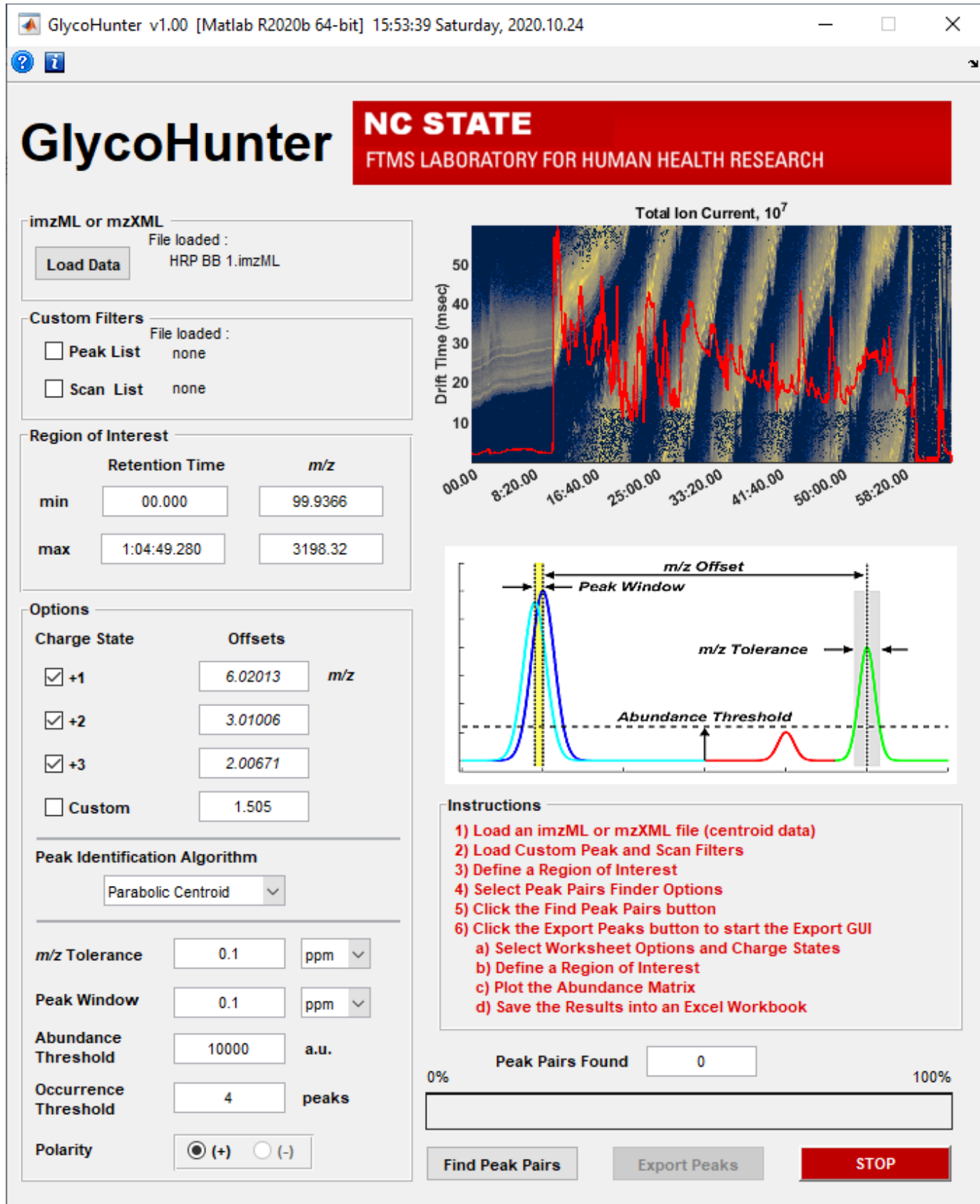

Prepared by: Ken Garrard and Jaclyn Kalmar 
Table of Contents

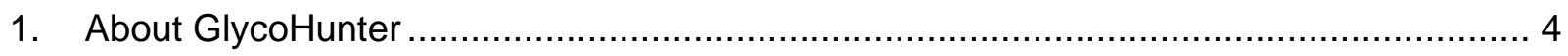

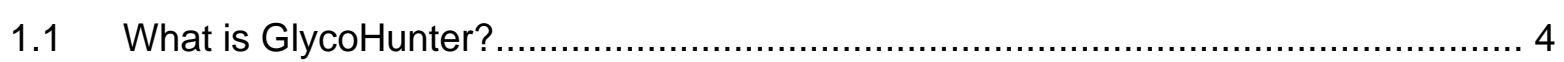

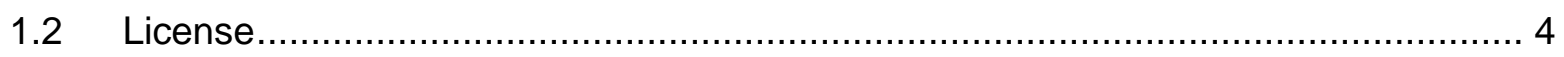

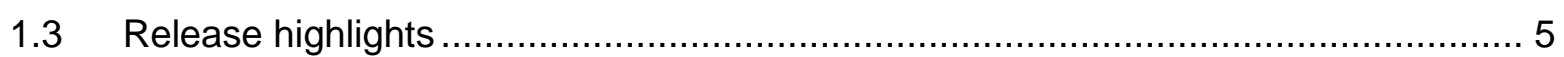

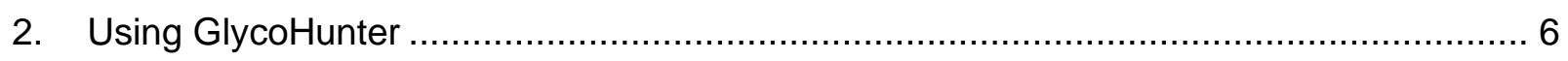

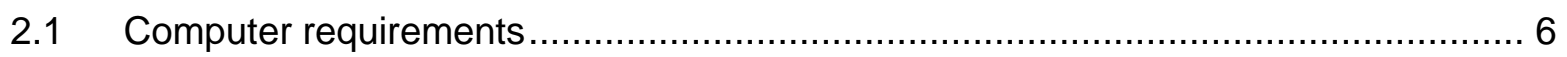

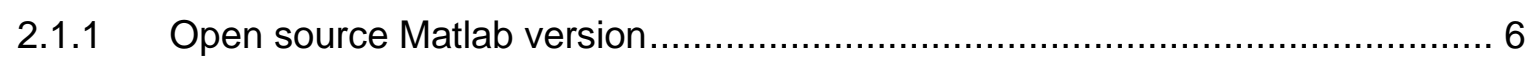

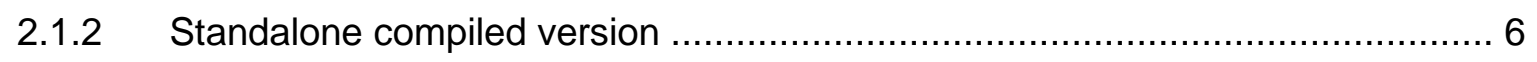

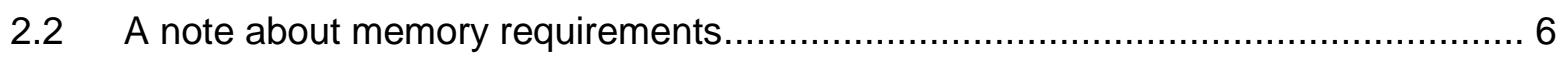

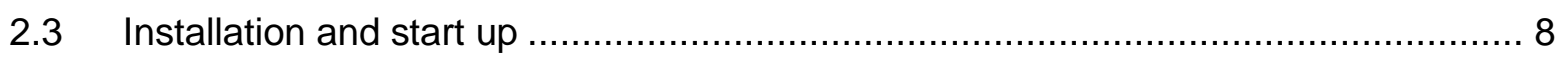

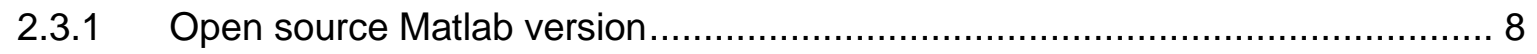

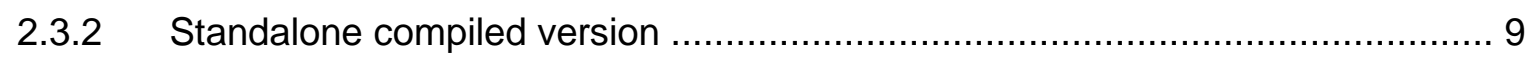

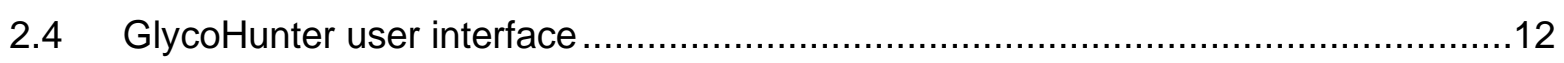

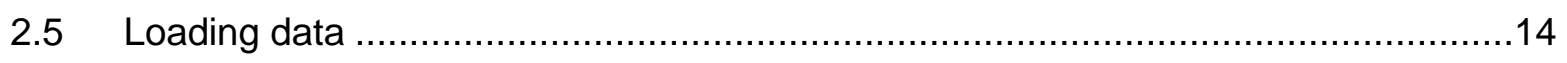

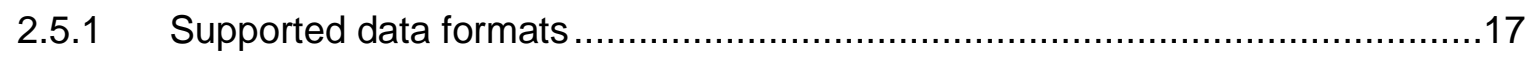

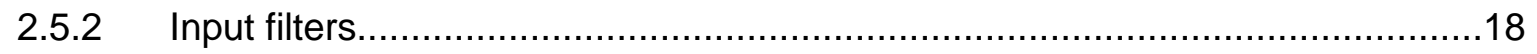

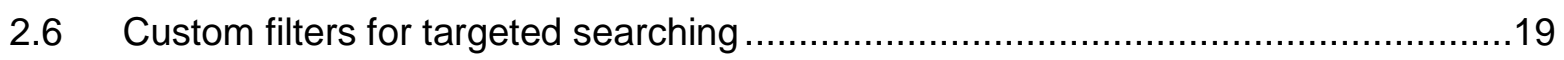

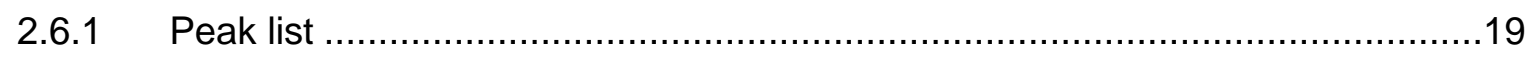

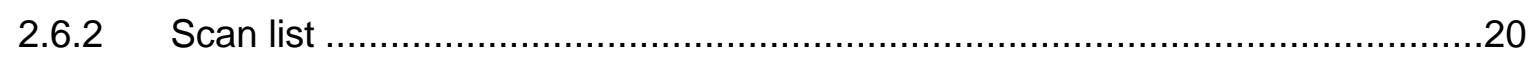

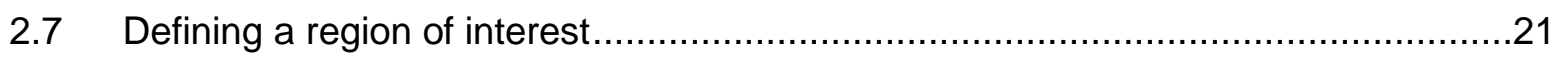

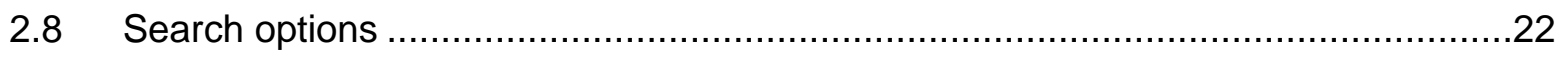

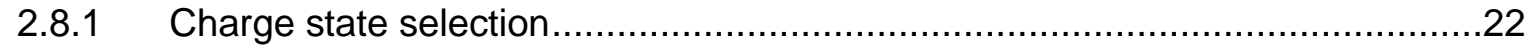

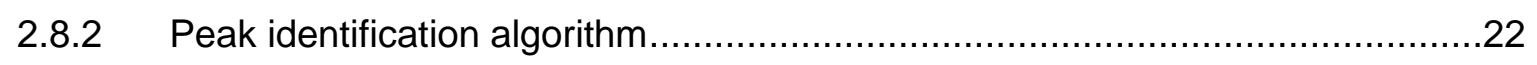

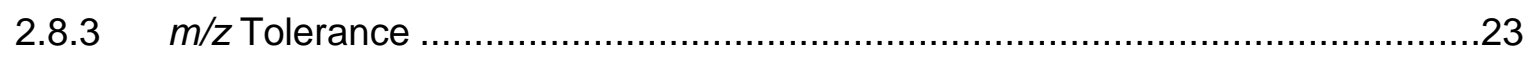

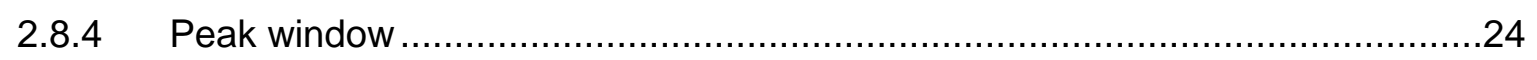

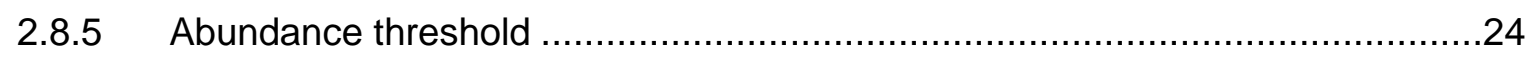

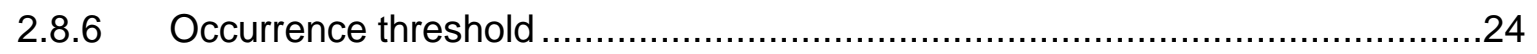

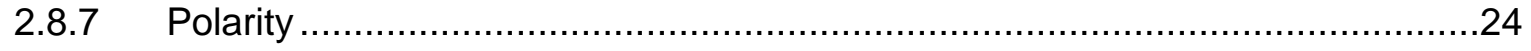

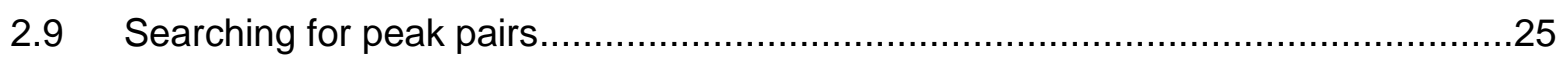

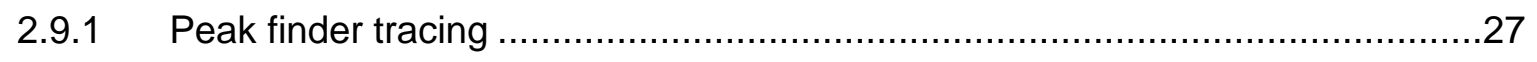

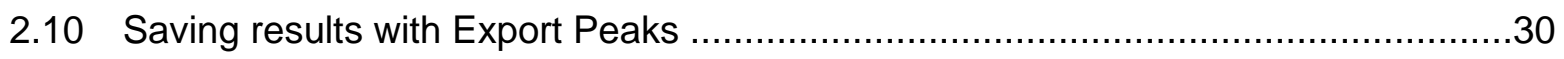

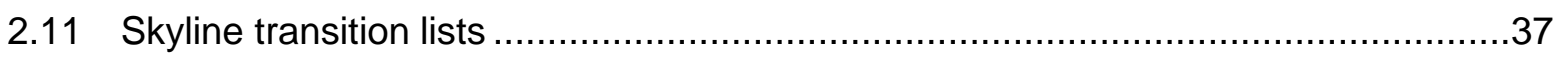

3. Customizing GlycoHunter - the preferences INI file ........................................42

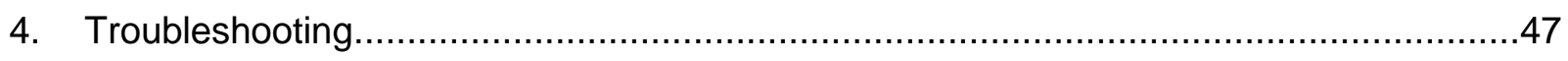

4.1 I get a "Java heap size" error message in the command window.......................47 


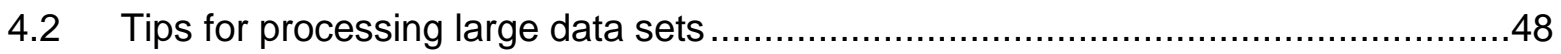

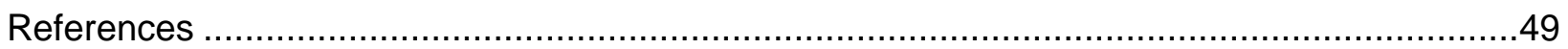




\section{About GlycoHunter}

\subsection{What is GlycoHunter?}

GlycoHunter is a free Matlab application designed to identity and perform relative quantification of INLIGHT TM labelled N-linked glycans. GlycoHunter performs both targeted and untargeted searches of MS1 data by finding peak pairs consisting of a monoisotopic value and one of up to four user defined $m / z$ offsets. GlycoHunter can read $m z X M L$ or imzML files and has limited support for ion mobility data. A sample imzML data set along with saved results files are included to test GlycoHunter and troubleshoot installation problems. Please contact us through the GlycoHunter website, glycohunter.wordpress.ncsu.edu, for suggestions, concerns, or to report problems.

\section{$1.2 \quad$ License}

GlycoHunter is released under the BSD 3 open source license [1]. This license allows you to use and modify the source code provided that the original copyright notice is included. The contents of the LICENSE.TXT file are shown below. It can also be found in the GlycoHunter installation folder.

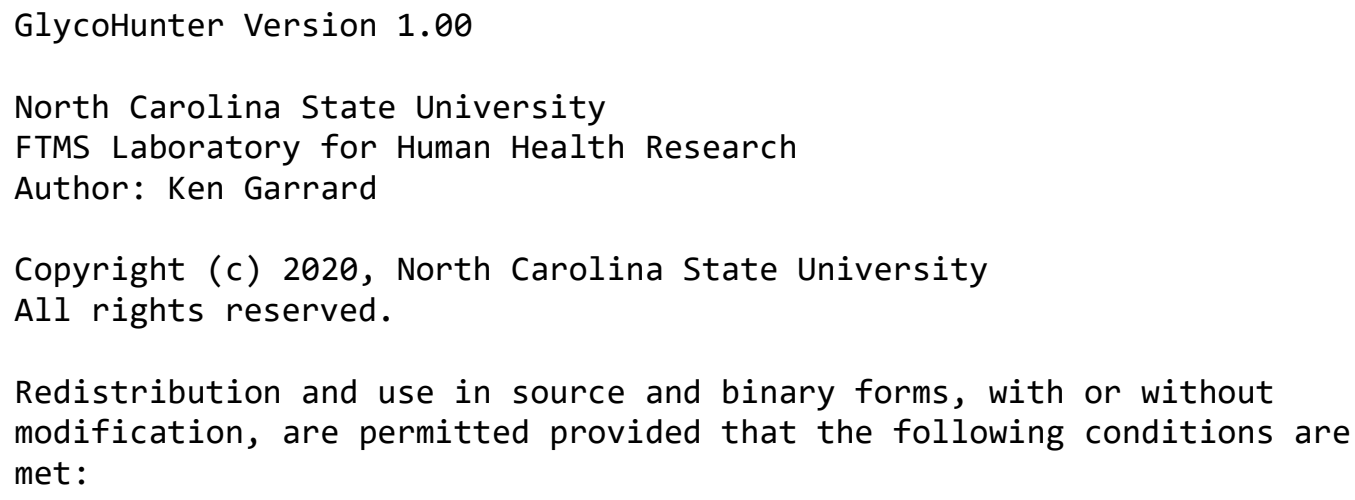

(1) Redistributions of source code must retain the above copyright notice, this list of conditions and the following disclaimer.

(2) Redistributions in binary form must reproduce the above copyright notice, this list of conditions and the following disclaimer in the documentation and/or other materials provided with the distribution.

(3) The names of the authors and copyright holder may not be used to endorse or promote products derived from this software without specific prior written permission.

THIS SOFTWARE IS PROVIDED BY THE AUTHOR ' AS IS'' AND ANY EXPRESS OR IMPLIED WARRANTIES, INCLUDING, BUT NOT LIMITED TO, THE IMPLIED WARRANTIES OF MERCHANTABILITY AND FITNESS FOR A PARTICULAR PURPOSE 
ARE DISCLAIMED. IN NO EVENT SHALL THE AUTHOR BE LIABLE FOR ANY DIRECT, INDIRECT, INCIDENTAL, SPECIAL, EXEMPLARY, OR CONSEQUENTIAL DAMAGES (INCLUDING, BUT NOT LIMITED TO, PROCUREMENT OF SUBSTITUTE GOODS OR SERVICES; LOSS OF USE, DATA, OR PROFITS; OR BUSINESS INTERRUPTION) HOWEVER CAUSED AND ON ANY THEORY OF LIABILITY, WHETHER IN CONTRACT, STRICT LIABILITY, OR TORT (INCLUDING NEGLIGENCE OR OTHERWISE) ARISING IN ANY WAY OUT OF THE USE OF THIS SOFTWARE, EVEN IF ADVISED OF THE POSSIBILITY OF SUCH DAMAGE.

\subsection{Release highlights}

Highlights of the initial public release of GlycoHunter are,

1. Load MS1 data from mzML or imzML format files.

2. Simultaneous peak pair searching for up to four $\mathrm{m} / \mathrm{z}$ offsets.

3. Search a region of interest (ROI) defined by retention time and $\mathrm{m} / \mathrm{z}$ ranges.

4. Targeted searching by $\mathrm{m} / \mathrm{z}$ and/or scan number.

5. Plot results as an abundance heatmap or in $3 \mathrm{D}$ as retention time vs $m / z$ vs abundance.

6. Export search results to Excel.

7. Export peak pairs to Skyline transition list format (CSV). 


\section{Using GlycoHunter}

GlycoHunter can be downloaded from glycohunter.wordpress.ncsu.edu. Both an open source version and a standalone, compiled version that can be used without a licensed Matlab installation are available. The standalone version of GlycoHunter 1.00 requires the Matlab compiler runtime (MCR) library for Release 2020b (version 9.9). Section 2.3 contains installation instructions for both versions.

\subsection{Computer requirements}

\subsubsection{Open source Matlab version}

The open source version of GlycoHunter has been tested with Matlab R2017a through R2020b under the Windows WIN7 and WIN10 64-bit operating systems. Excel is also required for exporting search results and abundance matrices to workbooks. Skyline transition lists are saved as CSV files and do not require Excel. In addition to the standard Matlab installation, the following Matlab toolboxes must be installed for all features of GlycoHunter to work properly.

- Bioinformatics

- Image processing

- Statistics and Machine Learning

\subsubsection{Standalone compiled version}

The standalone version can be used on computers where licensed Matlab software and the required toolboxes are not installed. It has been tested on Windows WIN7 and WIN10 64-bit computers. The standalone version uses the Matlab Compiler Runtime (MCR) for R2020b which is freely available from the Mathworks website. See Section 2.3.2 for detailed installation instructions. Excel is also needed to save search results. Skyline transition lists are saved as CSV files and do not require Excel.

\subsection{A note about memory requirements}

Predicting the maximum size data set GlycoHunter can process is not trivial since it depends on the amount of RAM available, which depends on the number of applications running on the computer. There needs to be enough RAM to store the data set and sufficient memory left for additional processing operations. Some of these may require the creation and temporary storage of large variables that depend on the search tolerances being used and the number of peak pairs found. However, the loaded data file almost always consumes most of the RAM used by GlycoHunter. An example memory usage calculation follows. 
Using a Windows 10 computer (Lenovo T470, Intel i5-7200, 2.5GHz with 24 GB of RAM), Matlab R2020b consumes about $1.8 \mathrm{~GB}$ and GlycoHunter requires approximately $400 \mathrm{MB}$ before a data set is loaded. Windows uses about $2 \mathrm{~GB}$, leaving $19.8 \mathrm{~GB}$ for data and additional code (e.g., the Export Peaks GUI, the Excel COM server). A single data point in a scan $(\mathrm{m} / \mathrm{z}$ value and abundance) uses 16 bytes of memory plus overhead. The overhead is proportional to the number of scans and ranges from 0.5 to 3 bytes per point for MS1 data and can be up to 16 bytes per point for sparse IM data since the spectra for each drift time are saved as separate scans. For example, if an MS1 data set with 50,000 scans is read and each spectrum contains an average of $10,000 \mathrm{~m} / \mathrm{z}$ data points, the memory requirement can be estimated as $50000 \times$ $10000 \times 18=9$ GB. Peak pair searching and data export requires no more than an additional $500 \mathrm{MB}$ even if $10 \mathrm{M}$ peak pair occurrences are found and the results exceed the limits of Excel. In case the data file is too large to be processed by your computer please see Section 4.2 . 


\subsection{Installation and start up}

\subsubsection{Open source Matlab version}

Here are step by step instructions for installing and opening GlycoHunter in the Windows environment using Matlab R2017a or later. The file GlycoHunterRelease1_00.zip contains three files: GlycoHunterRelease1_00_opensource.zip, GlycoHunterRelease1_00_standalone.zip, and LICENSE.TXT.

1. Copy and extract the files from GlycoHunterRelease1_00_opensource.zip to a location of your choice, for example c:Iusers|ken|documents|MATLAB|glycohunter. Make sure you have administrator rights for the selected folder. Do not put GlycoHunter in the Windows Program Files folder. The default name of the folder created when you unzip the open source archive is GlycoHunterRelease1_00_opensource. You may rename it if you wish.

2. When a Matlab session is started, a window like the one presented in Figure $\mathbf{1}$ will appear. To run GlycoHunter, the folder chosen in step 1 must be added to the Matlab path. From the Matlab ribbon style tool strip interface, select the Home tab and click on Set Path ...

3. Browse to find the folder where you extracted the open source contents in step 1, click OK and then Save. You do not need to add sub-folders of GlycoHunter to the path.

4. Close the Set Path menu.

5. GlycoHunter can now be started by entering "GlycoHunter" at the Matlab command prompt and pressing enter. Alternatively, right-click on the file GlycoHunter.m in the installation folder and select run. Do not try to start GlycoHunter by running (or double-clicking) the GlycoHunter.fig file! Note that you can also create a shortcut to GlycoHunter.m and save it in any convenient location on your computer (e.g., the desktop or taskbar). You do not have to start Matlab first if running GlycoHunter using this alternative method, but you must select run from the context menu for your shortcut. Double-clicking the shortcut will start Matlab and send the source code file to Matlab's m-file editor instead of running it.

6. The default Java heap memory in Matlab is typically set too low to operate GlycoHunter reliably. Instructions to increase the heap size are given in Section 4.1.

If you have sufficient memory you can start GlycoHunter multiple times in the same or different Matlab sessions and process multiple data sets simultaneously. Multiple Matlab sessions are normally run in different CPU cores. 


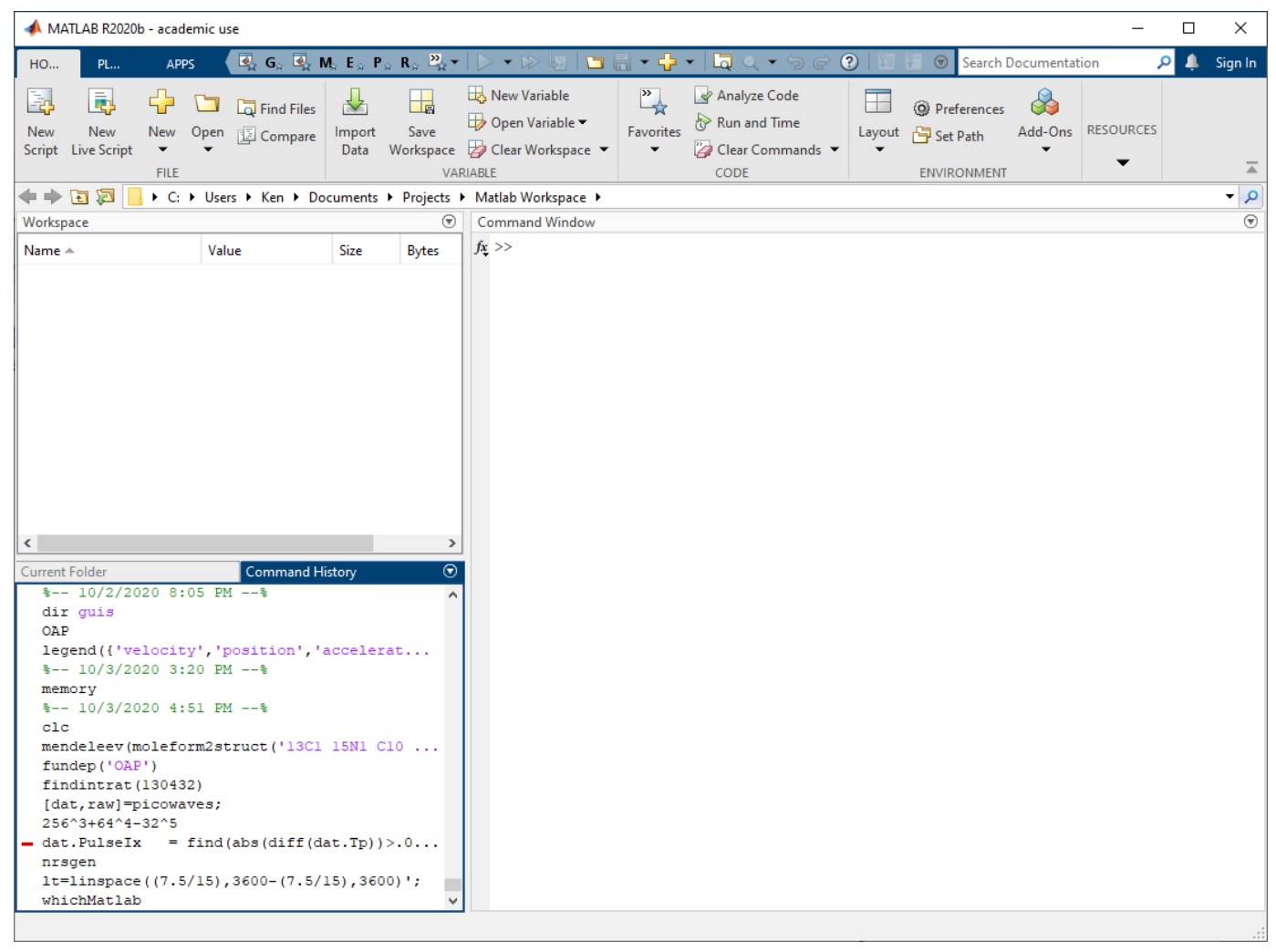

Figure 1. Matlab session.

\subsubsection{Standalone compiled version}

For deploying GlycoHunter to a computer without Matlab, the Matlab compiler in Release 2020b was used to produce an archive named GlycoHunterRelease1_00_standalone.zip. Step-by-step instructions to install and use this version are given below.

1. You will need to install the Matlab Compiler Runtime (MCR) for Matlab Release 2020b on your computer. The MCR is free and can be downloaded from the MathWorks website. Download the Windows 64-bit version of the MCR for Matlab R2020b (9.9) by navigating to,

http://www.mathworks.com/products/compiler/mcr/index.html

and selecting the file MATLAB_Runtime_R2020b_win64.zip.

2. Unzip the file downloaded in step 1 and install the MCR by running setup.exe. NOTE: You will need administrator rights to run this program.

3. Store the GlycoHunter application in a folder of your choosing by expanding the archive, 
GlycoHunterRelease1_00_standalone.zip

4. Always start GlycoHunter by executing the batch file, GlycoHunter_startup.bat.

For easy access, you may make a shortcut to the GlycoHunter_startup.bat file on your desktop or in a convenient folder. You can also pin the shortcut to the start menu or the taskbar. DO NOT copy the batch file to another location or move any of the other files! It is used to set an environment variable named "GLYCOHUNTER_PATH" to the application folder so that GlycoHunter can find its help files, templates, icons, etc. If you are comfortable setting persistent Windows environment variables using the System control panel then you can set the path permanently and use the GlycoHunter.exe file or a shortcut to it to start GlycoHunter.

When you run GlycoHunter_startup.bat, a command window will open for the duration of your GlycoHunter session. You can minimize it to the taskbar, but DO NOT close it or Windows will terminate GlycoHunter without a confirmation dialog or any other warning. Progress messages will appear in this command window when a file is loaded, peak pair searching is performed, results are saved, etc. If you see error messages from Windows or Matlab other than those shown below in Figure 2 please send a screenshot (Alt-PrtSc) to glycohunter@gmail.com. When GlycoHunter terminates, the command window will remain open for 4 seconds to allow a screen capture of its contents in the event of an error that has crashed GlycoHunter.

Note 1 - Windows security scan. Depending on your Windows Defender or other virus and malware software settings there may be an additional delay for a virus scan to complete the first time the glycohunter.exe file is executed. This is enabled by the Microsoft Defender ATP "block at first sight" feature and is usually accompanied by a Windows notification.

Note 2-GlycoHunter fails to terminate. On some Windows 7 installations after the user terminates GlycoHunter a Matlab MCR bug prevents the command window from automatically closing. If this happens closing the command window manually completes the shutdown of GlycoHunter.

Note 3 - GlycoHunter fails to terminate. On managed Windows 10 installations there is a known issue with the Google Drive File Stream error management system. This is not specific to GlycoHunter or deployed Matlab applications. The first time a file is loaded into GlycoHunter error messages similar to those shown in Figure 2 may appear. GlycoHunter loads the file and runs normally. However, you may need to manually close the command window after terminating GlycoHunter. 


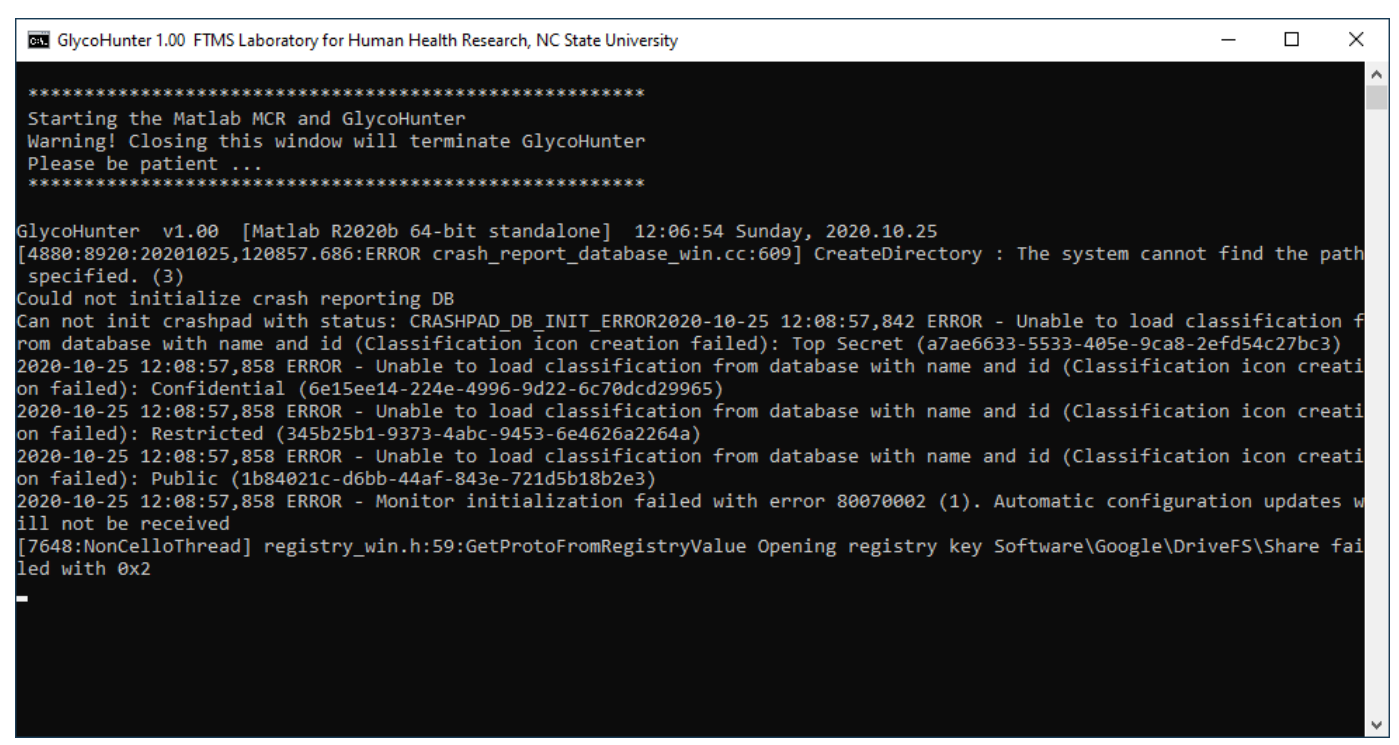

Figure 2. Standalone session error messages from Google drive file stream.

Please be patient waiting for standalone GlycoHunter to start. The Matlab runtime must start first and no progress information is displayed. After the first run, the Matlab runtime will start faster until a system cleanup utility is run. If you have sufficient memory you can run multiple instances of the standalone GlycoHunter by running the startup batch file more than once.

Note 4 - Networked drive installation. If GlycoHunter is installed on a remote, networked disk you should assign the shared space a drive letter. The Matlab MCR will not load properly if the startup batch file is launched via a Universal Naming Convention (UNC) path.

Note 5 - Installation on portable storage media. You can save the GlycoHunter installation on an external hard drive or SSD if you do not have sufficient space on your boot drive or in your user account folders. However the MCR is installed in the Program Files folder with other applications and it needs a substantial amount of space (> $6 \mathrm{~GB}$ ) on the same drive as the operating system to work properly. It is essentially all of Matlab without a user interface or program files editor. If the standalone GlycoHunter crashes with MCR related error messages in the command window, try freeing up space on the boot drive. 


\subsection{GlycoHunter user interface}

GlycoHunter is programmed in Matlab using the MathWorks graphical user interface design environment (GUIDE). It consists of a window for the main interface as shown in Figure $\mathbf{3}$ and one sub-GUI for exporting search results (See Section 2.10). Interface objects are menus, push buttons, toggle buttons, edit boxes, popup menus, listboxes, sliders, toolbars, dialog and message boxes, checkboxes and mutually exclusive radio button groups. GlycoHunter and the Export Peaks sub-GUI have no menus and are driven primarily with edit boxes for user defined parameters, popup menus, and push button controls. Arithmetic expressions that are typed into edit boxes for $\mathrm{m} / \mathrm{z}$ values, tolerances, and thresholds are evaluated when the enter key is pressed.

Both GUI windows and exported plot figures have a title bar that includes the version of GlycoHunter or Export Peaks, the version of Matlab and the date and time. The icon toolbar if any is under the title. Exported figures inherit the standard Matlab toolbars and menus and include features to edit and annotate the plot, pan and zoom, save and print. Some of the Matlab figure editing functions are not available for the standalone version.

The GlycoHunter GUI has two icons on its toolbar.

Launch the default PDF viewer on your system and load this manual.

i Display license and copyright information in a message box.

Several important features are accessed with right-click context menus on buttons, panels, checkboxes, and edit boxes. These are,

1. Removing a peaks list filter (Section 2.6.1),

2. Copying the $m / z$ values in the peaks list filter to the clipboard (Section 2.6.1),

3. Changing the peaks list filter tolerance (Section 2.6.1),

4. Removing a scan list filter (Section 2.6.2),

5. Copying the scan numbers in the scan filter to the clipboard (Section 2.6.2),

6. Resetting the STOP button,

7. Resetting the Status bar,

8. Resetting the ROI minimum and maximum retention time in Export Peaks (Section 2.10),

9. Resetting the ROI minimum and maximum $\mathrm{m} / \mathrm{z}$ in Export Peaks (Section 2.10). 


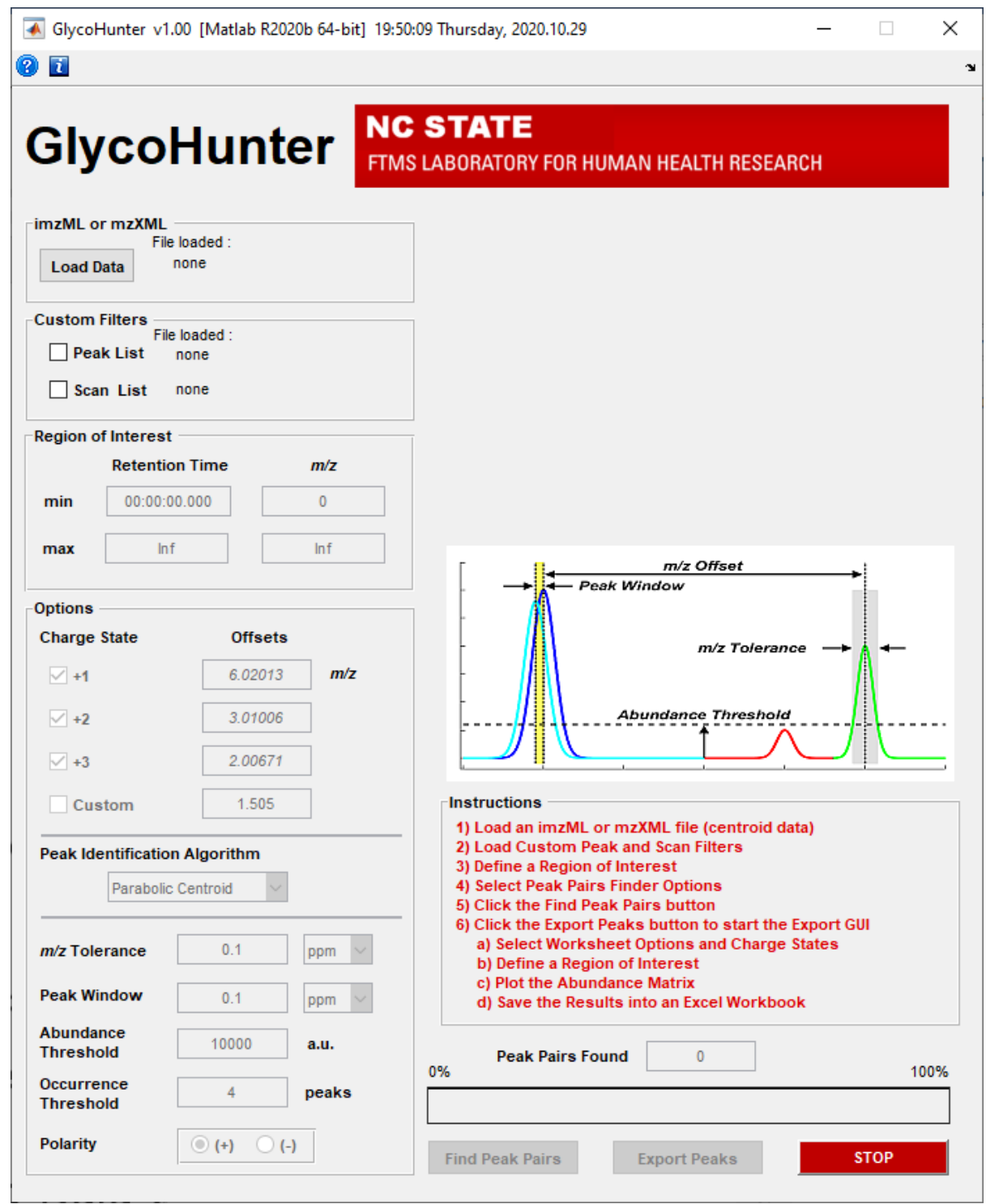

Figure 3. The GlycoHunter user interface immediately after startup.

On the right in Figure 3 the meaning of the search options is illustrated and a synopsis of a typical workflow is given in the Instructions panel. The space above the illustration is reserved for a TIC plot of the loaded data set. 


\subsection{Loading data}

After starting GlycoHunter, a data set can be loaded by clicking on the Load Data button in the upper left panel of the user interface. A standard Windows file selection dialog is launched for the user to select a data set. The starting path for the selection can be specified with a variable in the preferences INI file (See Section 3). Either an mzXML or imzML file can be loaded by changing the file type selection in the dialog shown in Figure 4.

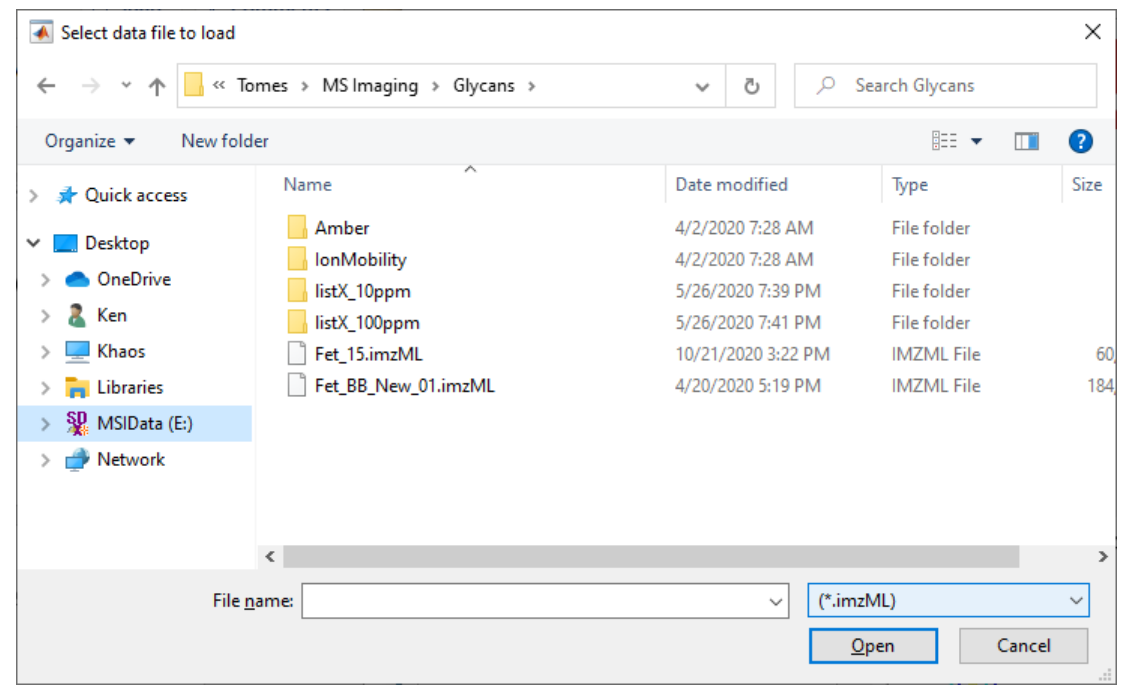

Figure 4. The GlycoHunter file selection dialog.

After a file is selected GlycoHunter begins loading the data. For imzML data sets, GlycoHunter parses the imzML file and saves the results in a Matlab binary MAT file in the same folder and with the same name as the data being loading. The file extension of this file is ibh. Subsequently loading the file is as much as 10 times faster using this preprocessed version of the imzML metadata. As shown in Figure $\mathbf{5}$ the status bar updates proportionally as scans are loaded. Clicking the STOP button will launch the dialog shown in Figure 6 where the user can either cancel or resume loading the data. 


\section{GlycoHunter}

NC STATE

FTMS LABORATORY FOR HUMAN HEALTH RESEARCH

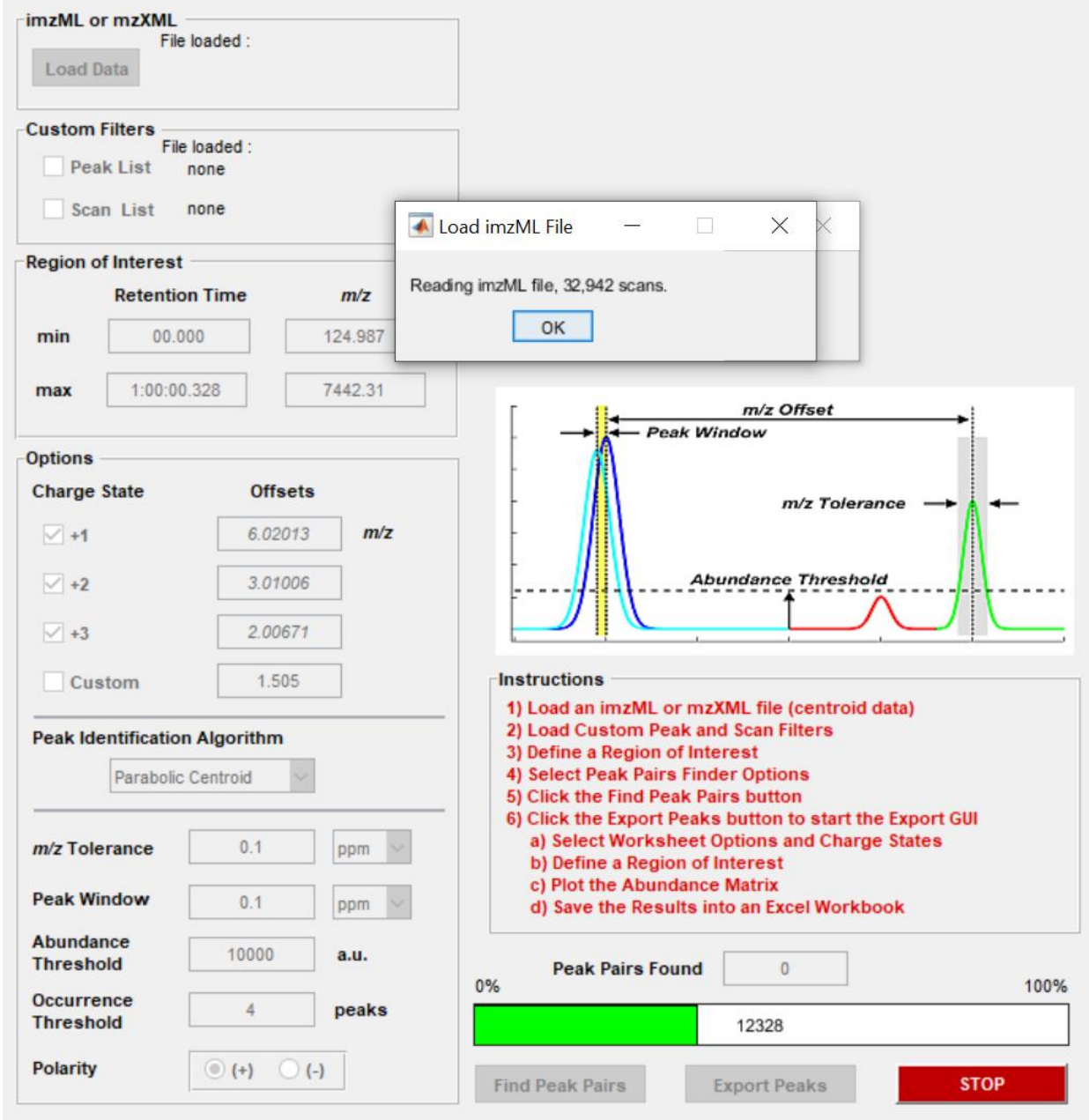

Figure 5. The GlycoHunter status bar indicates progress while loading a data set.

Cancel

Are you sure you want to cancel loading the data set?

Yes, Cancel No, Continue

Figure 6. The GlycoHunter cancel/continue dialog. 
When all scans are loaded a total ion current plot is displayed in the upper right portion of the user interface window as shown in Figure 7 . If the data set (imzML format only) contains ion mobility data, the TIC plot is overlaid on top of a drift time vs retention time plot as shown on the front cover of this manual. Custom filters, a region of interest, and search options are now enabled for the user to initiate a peak pairs search.

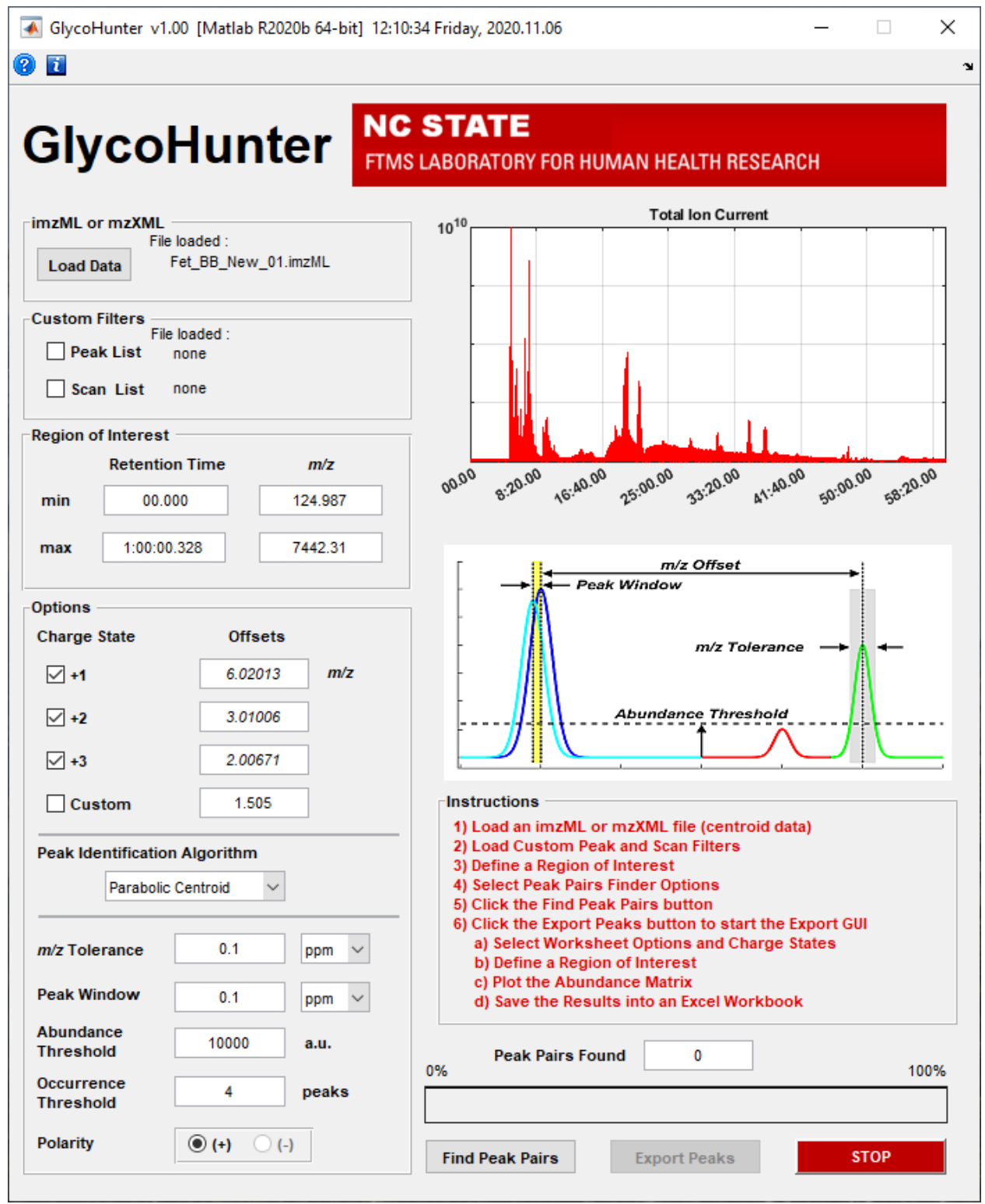

Figure 7. GlycoHunter after loading a data set. 


\subsubsection{Supported data formats}

Steps to load each file type are described below.

1. imzML File This file format was developed by the EU project Computis $[2,3]$ to efficiently store and share imaging mass spectrometric data; however the data need not be from a spatial image to be converted to this format. It is the preferred format for GlycoHunter since it contains the required meta-information, it is compact, it loads much faster than the $m z X M L$, and data from a variety of instrument vendors can be converted to imzML. An imzML data set consists of two files, one XML file (imzML extension) that contains the metadata such as the instrument parameters and the scan pattern information and a binary file (ibd extension) that contains the mass spectrometric data. There are multiple converters that can translate directly from a vendor's format to imzML (e.g. RawtolmzmlConverter (Thermo Scientific), HDI (Waters), etc.). The http://www.maldi-msi.org website maintains upto-date versions of these converters in their imzML section. Users can also use MSConvert from Proteowizard [4] to convert vendor's format to HUPO-PSI's mzML [5] and then use imzMLConverter [6] to convert the mzML file to imzML format. Recently a user-friendly validation and editing tool, the imzMLValidator has become available [7] to ensure that converted files conform to the standard. It can be downloaded here [8]. Please use the validator if you have problems loading your data. It includes tools to repair incorrect metadata and to add missing metadata. ImzML files can be generated in processed (one $\mathrm{m} / \mathrm{z}$ array per scan) or continuous mode (one $\mathrm{m} / \mathrm{z}$ array per file). Either format can be loaded into GlycoHunter, but the user must be careful to choose the appropriate format for the type of instrument used when converting a file to imzML. For example, scan data from a RAW file collected on a Thermo Scientific FTICR are already in a processed format and should therefore not be converted to a continuous format. More information about continuous and processed files can be found in the literature cited above.

2. $m z X M L$ File $m z X M L$ is an open representation for MS data introduced by Pedrioli et Al. [9]. An mzXML file can be obtained from RAW data using the MSConvert tool from Proteowizard [5]. The mzXML format encodes spectral data into tagged XML records and is thus a less efficient use of storage space and is more time consuming to decode and load than imzML. 


\subsubsection{Input filters}

A data set can be filtered by both $\mathrm{m} / \mathrm{z}$ and abundance as it is loaded to conserve memory space and improve performance.

1. Abundance threshold Scan spectra in many data sets contain a large number of zero or very low abundance values. These $m / z$, abundance points can be filtered out of the data as the file is loaded by setting the MinAbundanceThreshold variable in the preference INI file (See Section 3). The threshold value is the smallest abundance that is retained for any data pair in a scan. The default value is 0.001 . It can be changed to any value between zero and 1 e9.

2. $m / z$ Range Scan can also be filtered by $m / z$ value as they are loaded. The $m z$ FilterMin and $m z F i l t e r M a x$ variables in the preferences INI file can be used to define an $m / z$ range. Data pairs outside of this range will not be saved as each scan is read. The default values are zero and Infinity. 


\subsection{Custom filters for targeted searching}

A targeted search for peak pairs matching the user's criteria can be performed by loading a list of $m / z$ values from an Excel or text file. Only peaks within a specified tolerance will be selected as an $\mathrm{m} / \mathrm{z}$ low value when searching for peak pairs. Similarly the search can be restricted to specific scans by loading a list of scan numbers from an Excel or text file.

\subsubsection{Peak list}

When the Peak List checkbox in the Custom Filters panel (Figure 7) is first checked the file selection dialog shown in Figure $\mathbf{8}$ is displayed. The user may select either an Excel file or a text file. If the selected Excel file contain multiple worksheets the user is prompted to select one as shown in Figure 9 (left). If the selected worksheet contains more than one column the dialog in Figure 9 (right) is used to pick a column.

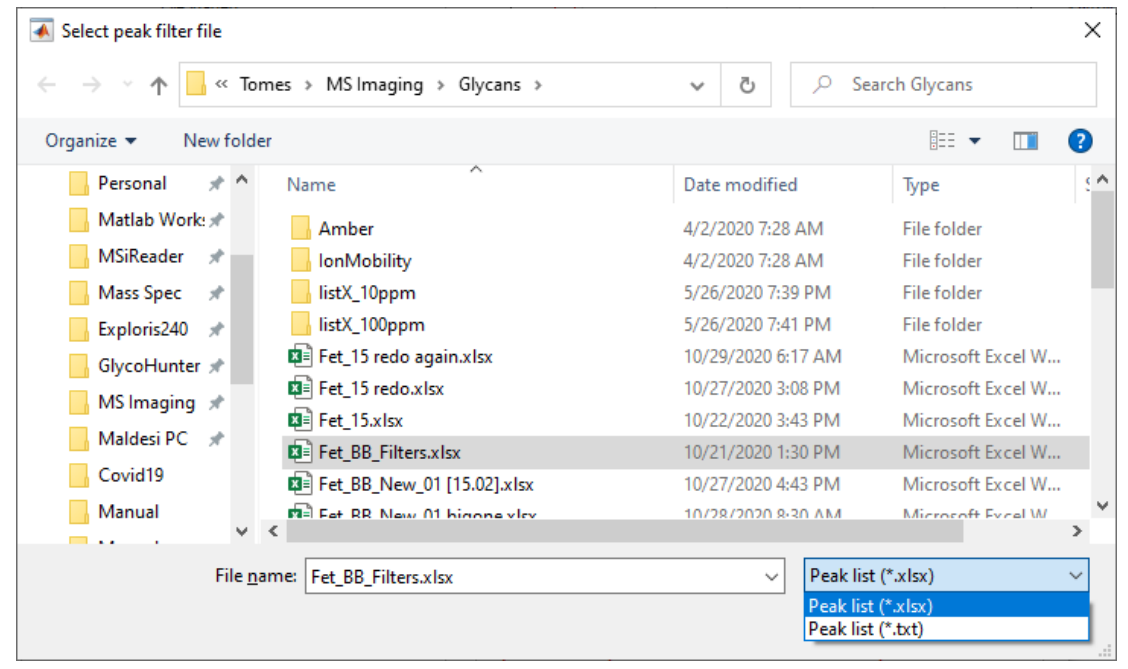

Figure 8. The custom peak filter selection dialog.
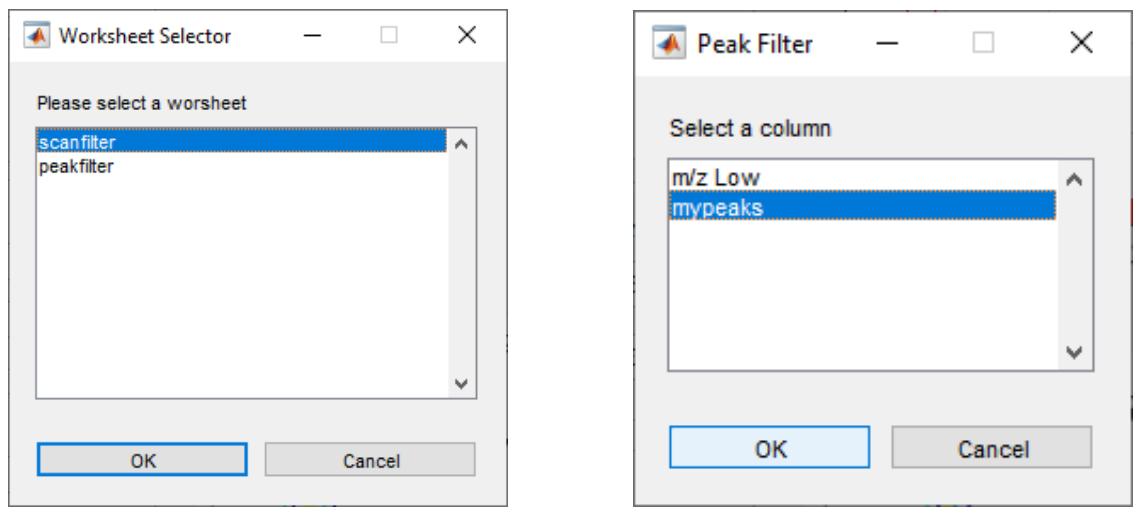

Figure 9. Worksheet (left) and column (right) selection dialogs for the peak list filter. 
If a text file is chosen with more than one column the user is prompted to select the column containing a list of $m / z$ values for the filter.

Unchecking the checkbox disables the filter but does not remove the filter $m / z$ values. The rightclick context menu for the Peak List checkbox shown in Figure $\mathbf{1 0}$ can be used to remove the peak filter so another file can be selected, to copy the peak list to the clipboard, and to edit the peak filter tolerance. The default peak filter tolerance is $1 \mathrm{ppm}$. This value can be changed by setting the PeakFilterTolerance value in the INI preferences file (See Section 3).

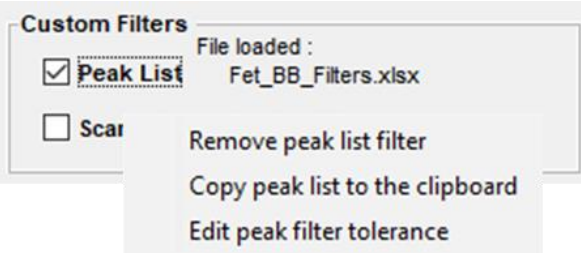

Figure 10. Peak list context menu selections.

\subsubsection{Scan list}

Peak pairs searching can also be restricted to a specific list of scan numbers using the Scan List filter in the Custom Filters panel (Figure 7). Loading and enabling a scan filter is similar to a filter except that there is no tolerance specification in the context menu or the INI preference file. The user is prompted to select a file, a worksheet if there is more than one, and a column of numeric values if there is more than one. 


\section{7 $\quad$ Defining a region of interest}

Peaks pairs searching can also be restricted to a region of interest (ROI) in both retention time and $m / z$ space. The default ROI is the full range of time and $m / z$ in the loaded data set. This can be changed by typing new values in the Region of Interest panel shown in Figure 11. The ROI can be reset to the full range entering the values 0 and Inf. Hovering the mouse over either of the retention time values will pop-up a box with the time in hours, minutes, and seconds converted to seconds.

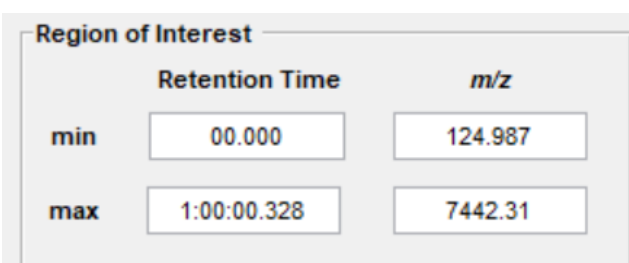

Figure 11. Defining a region of interest.

If peak and scan filters are defined in addition to an ROI, both are applied during the scan search. For example, a putative monoisotopic $m / z$ value must be within the ROI range and within the tolerance of some $\mathrm{m} / \mathrm{z}$ in the peak list filter. 


\subsection{Search options}

\subsubsection{Charge state selection}

Up to four $m / z$ offset values defining peak pairs can be defined and selected for searching. The default values correspond to charge states $+1,+2,+3$, and a custom value. Any positive number or arithmetic expression that gives a positive value can be entered in the offset edit boxes. The defaults values, checkbox states, and labels can be changed in the INI preferences file. See (Section 3). Labels defined in the INI file will be used in the GlycoHunter and Export Peaks user interface windows and in exported Excel worksheets and abundance matrix plots.

\subsubsection{Peak identification algorithm}

Three different algorithms can be used to extract a peak list from the spectrum data. The algorithms use different approaches to calculate the centroid. The default option is to use a Parabolic Centroid Algorithm. This algorithm was proposed by Comisarow and Marshall in their early work interpreting FTMS spectra [10,11]. For each local maximum in a mass spectrum, the centroid location and abundance will be the calculated $\mathrm{m} / \mathrm{z}$ of the apex of a parabola fit to that local maximum and the 2 adjacent points (see Figure 12). The centroid locations calculated with this method are nearly identical (within a fraction of ppm) to those calculated by Xcalibur (Thermo Scientific) on their LTQ FT-ICR and Orbitrap instruments.

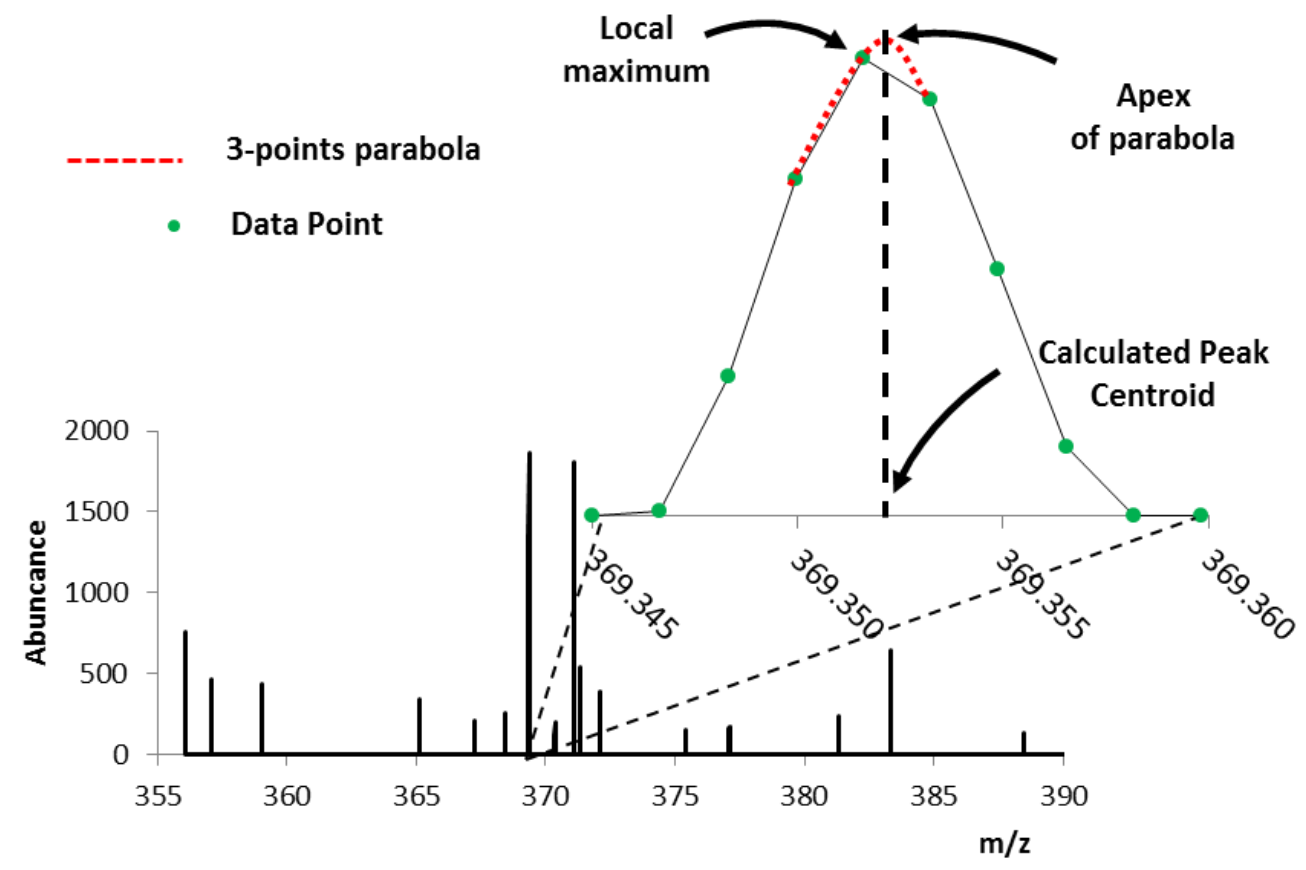

Figure 12. Calculated peak location using the parabolic centroid algorithm. 
The second algorithm calls the Matlab mspeaks function which is included in the Bioinformatics toolbox to calculate peak centroid locations using the discrete wavelet transform. More information about this function can be found on the Mathworks website [12] or by typing help mspeaks at the Matlab command prompt.

The third algorithm selects local maxima as candidate peaks in each scan. That is, the $m / z$ with the maximum abundance value in each local data window. This is of course the fastest of the three algorithms and ensures that selected peaks have $m / z$ values and abundances that are identical to data points in the original scan data. Local maxima should be used if the data has been centroided.

The default selection can be changed using the preferences INI file as described in Section 3. Also note that using mspeaks may increase computation time significantly.

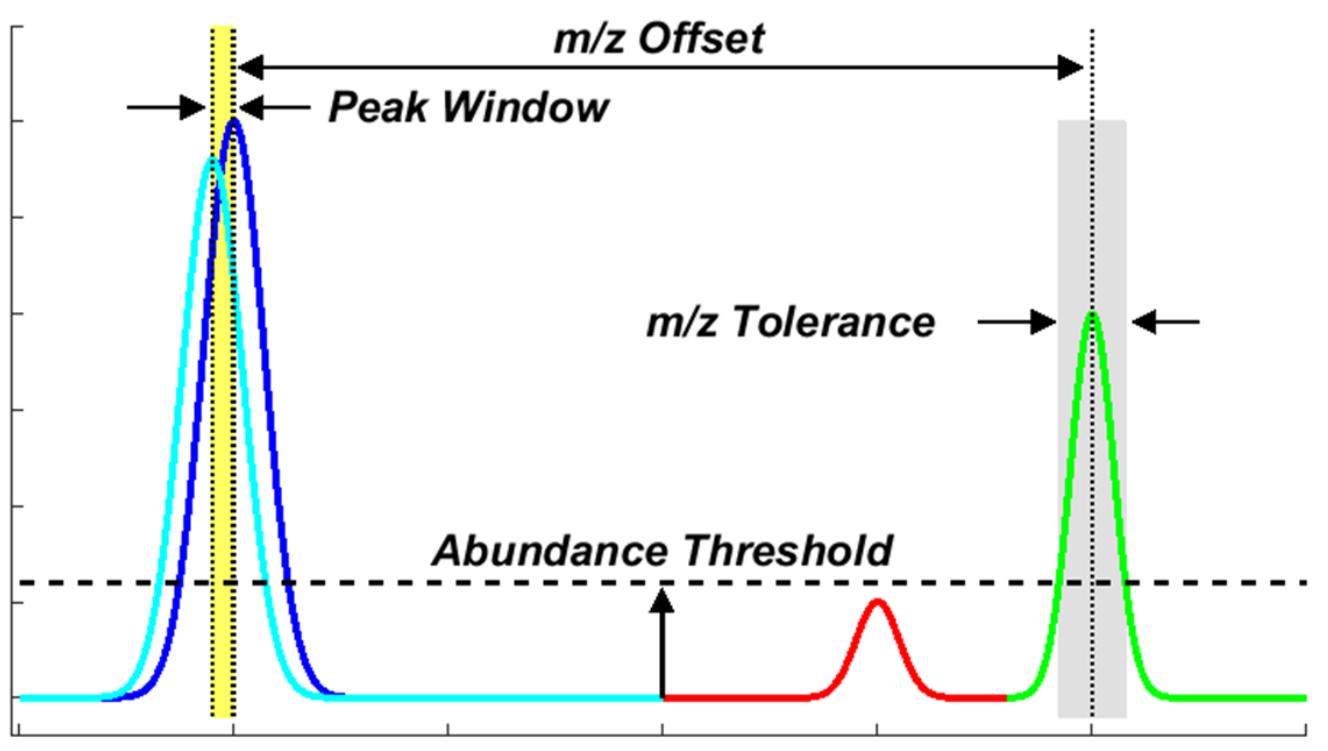

Figure 13. Peak pairs search parameters.

\subsection{3 $\mathrm{m} / \mathrm{z}$ Tolerance}

The $\mathrm{m} / \mathrm{z}$ tolerance is the range of deviation from the exact charge state offsets for any putatively identified peak pair. The units can be either ppm or $\mathrm{m} / \mathrm{z}$. This is illustrated by the gray shaded box in Figure 13. The default value is $0.1 \mathrm{ppm}$. The user should consider the instrument settings (e.g., resolving power and mass accuracy) used to acquire the data when specifying 
this parameter. In general, a larger tolerance will increase the number of peak pair occurrences found.

\subsubsection{Peak window}

The peak window is the range of $\mathrm{m} / \mathrm{z}$ considered when identifying unique peak pairs. In Figure 13 both the blue and cyan peaks are within the same window and would thus be considered as two occurrences of the same monoisotopic peak provided they have the same charge state match. A smaller peak window will usually increase the number of unique peak pairs identified by GlycoHunter since closely spaced peaks are less likely to be considered multiple occurrences of the same peak.

\subsubsection{Abundance threshold}

The abundance threshold is simply the lowest abundance (a.u.) that will be considered a peak by the centroiding algorithms discussed in Section 2.8.2.

\subsubsection{Occurrence threshold}

The occurrence threshold is the minimum number of times a peak pair must occur to be included in the final results.

\subsubsection{Polarity}

For data sets containing both positive and negative polarity scans, a polarity must be selected for each search. 


\subsection{Searching for peak pairs}

After the custom filters, region of interest, and search options have been selected the search for peak pairs matching the search criteria is initiated by clicking the Find Peak Pairs button. A dialog will be displayed and the status bar will track the search progress by displaying the current scan and its retention time as shown in Figure 14. The number of putative peak pairs found so far is also displayed.

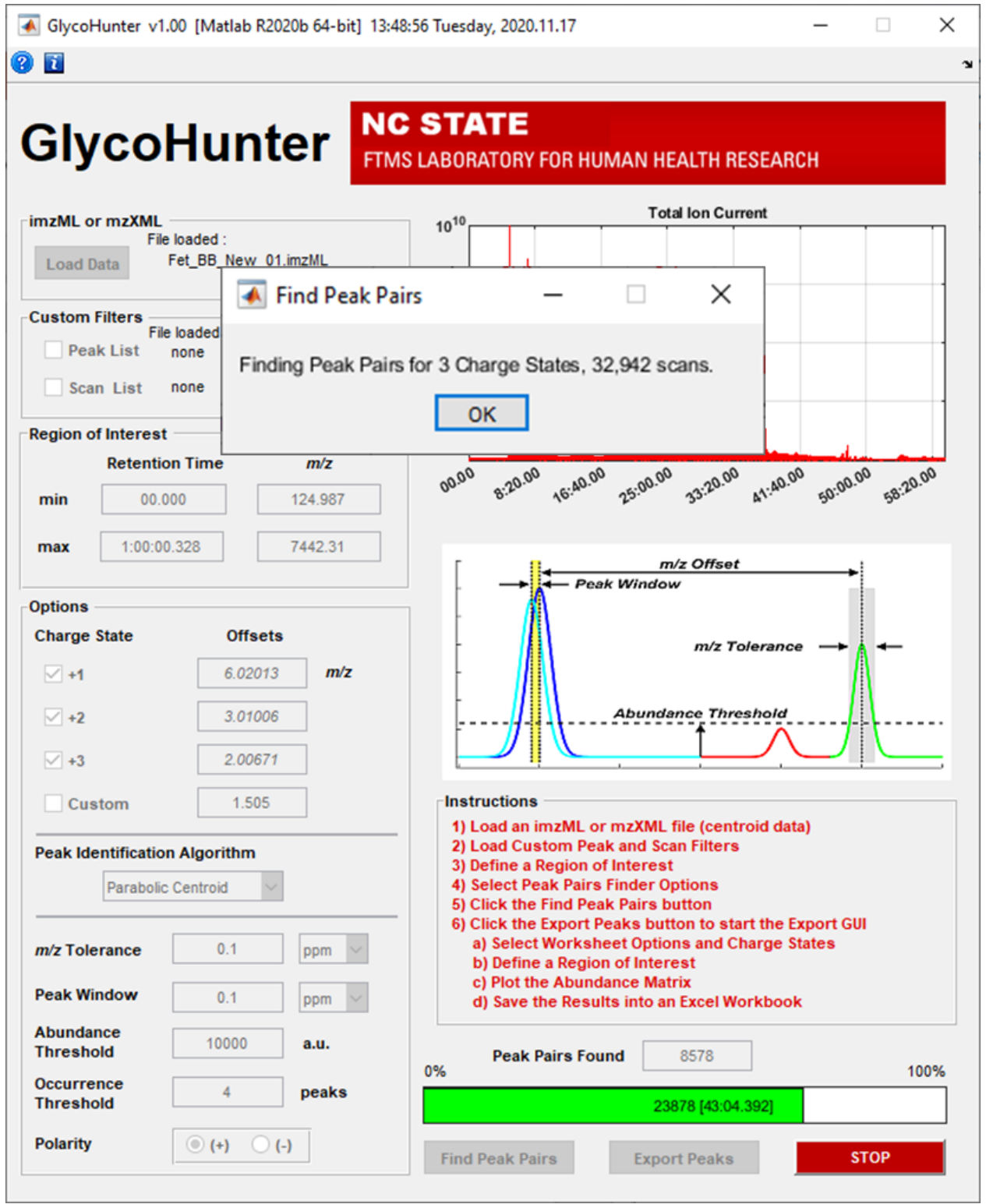

Figure 14. Searching for peak pairs. 
The STOP button can be pressed at any time to terminate the search. The dialog show in Figure 15 (left) gives the user the option to continue the search or to cancel it. If canceled, the search results found so far are retained and can be processed by the Export Peaks GUI. When a search is completed or canceled a dialog similar to Figure 15 (right) will be displayed containing a summary of the results.
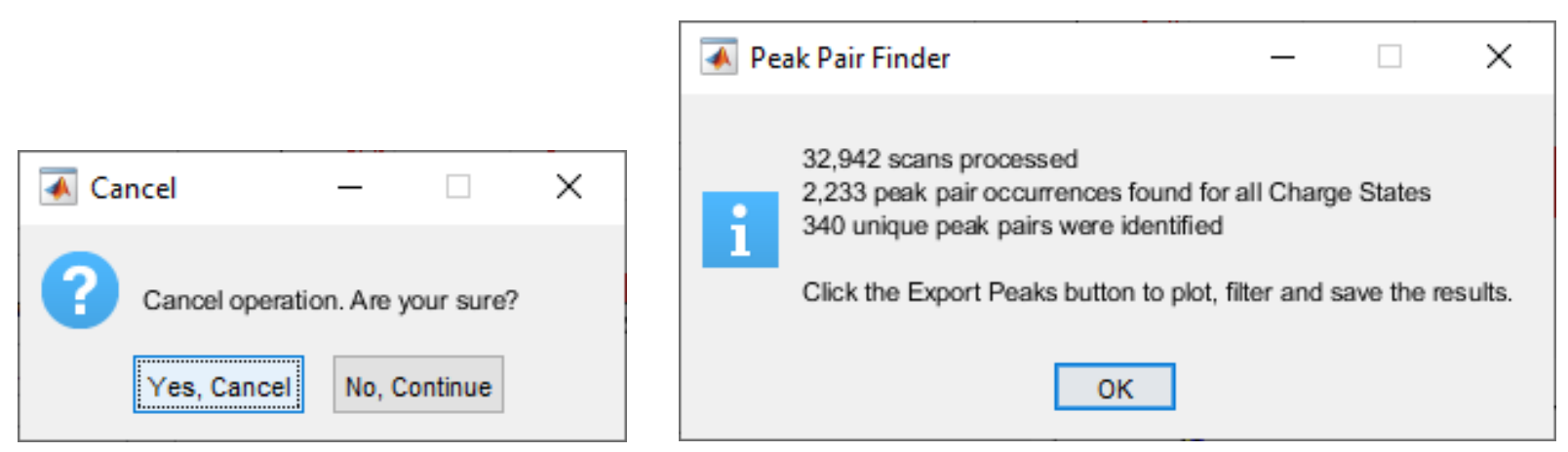

Figure 15. Peak pair search dialogs: Cancel/continue (left), Completion summary (right).

The search algorithm iterates on each scan and for each selected charge state, saving each pair of peaks that match the search criteria in an internal data structure. When the search terminates the Peak Window parameter is used to group multiple occurrences of the same pair. The results are then filtered by removing peak pairs whose occurrence count is below the threshold parameter. 


\subsubsection{Peak finder tracing}

GlycoHunter has two peak finder tracing features that can be enabled using preferences INI variables (See Section 3). If the preferences INI variable PeakFinderTrace is set to true a message will be written to the command window every time a potential peak pair is found. This will slow the search function significantly if there are many thousands of peaks but may aid with debugging problems. A portion of this output is shown below in Figure 16 after being pasted into a Notepad text file. The Peakldx and Mateldx columns refer to the index of the data points in the raw scan data vector that forms a peak pair matching the search criteria.

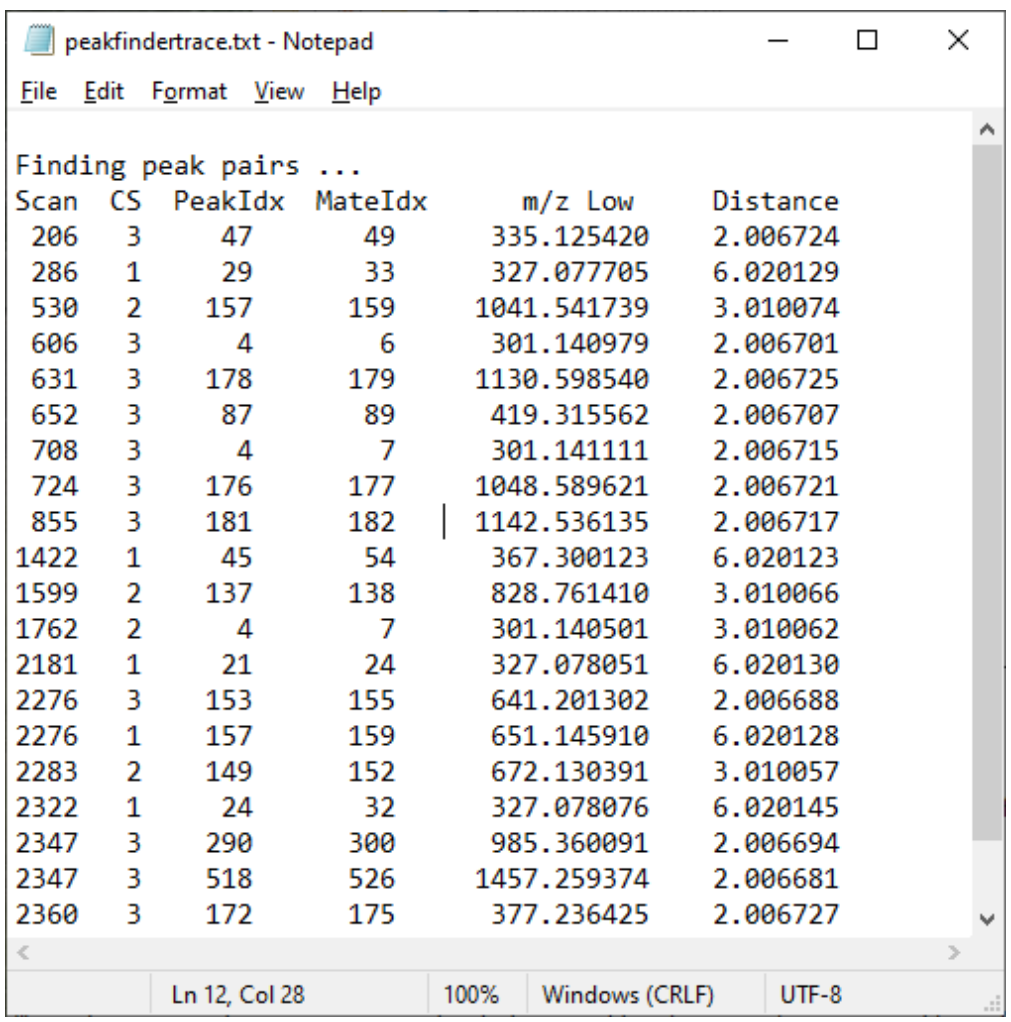

Figure 16. Peak finder trace.

A more detailed trace of search progress for a single target $m / z$ value can be enabled with the mzTargetTrace, mzTarget, and mzTargetTolerance preferences INI variables. Search results for every scan will be displayed in the command window whether or not a peak was found within the tolerance window. The mzTargetTolerance has units of ppm. Setting mzTargetTrace to a value other than zero enables this feature. The allowed values and their meaning are given in Table 1. 
Table 1. Peak target trace options.

\begin{tabular}{|c|l|}
\hline $\boldsymbol{m z}$ TargetTrace & Meaning \\
\hline 0 & Disable $\mathrm{m} / \mathrm{z}$ target tracing \\
\hline 1 & Print messages to the command window \\
\hline 2 & $\begin{array}{l}\text { Print message to the command window } \\
\text { and plot scans in realtime }\end{array}$ \\
\hline 3 & $\begin{array}{l}\text { Print messages to the command window } \\
\text { and plot results for each putative peak }\end{array}$ \\
\hline 4 & $\begin{array}{l}\text { Print messages to the command window } \\
\text { and plot results for each scan in a separate plot }\end{array}$ \\
\hline
\end{tabular}

Care should be taken when enabling peak target tracing and a narrow region of interest should be defined. In particular, option 4 will create a new plot for every scan in the search ROI and option 3 will create one for each putative peak pair. Defining a narrow ROI around the peak of interest will likely make the plots more meaningful. A portion of a command window output using option 3 is shown in Figure 17 and an example of the plot for a scan is shown in Figure 18. The plot has a vertical red line at the target $\mathrm{m} / \mathrm{z}$ and red crosses for every peak. The command window messages have a list of peaks for each scan with the ppm distance to the one closest to the target $m / z$.

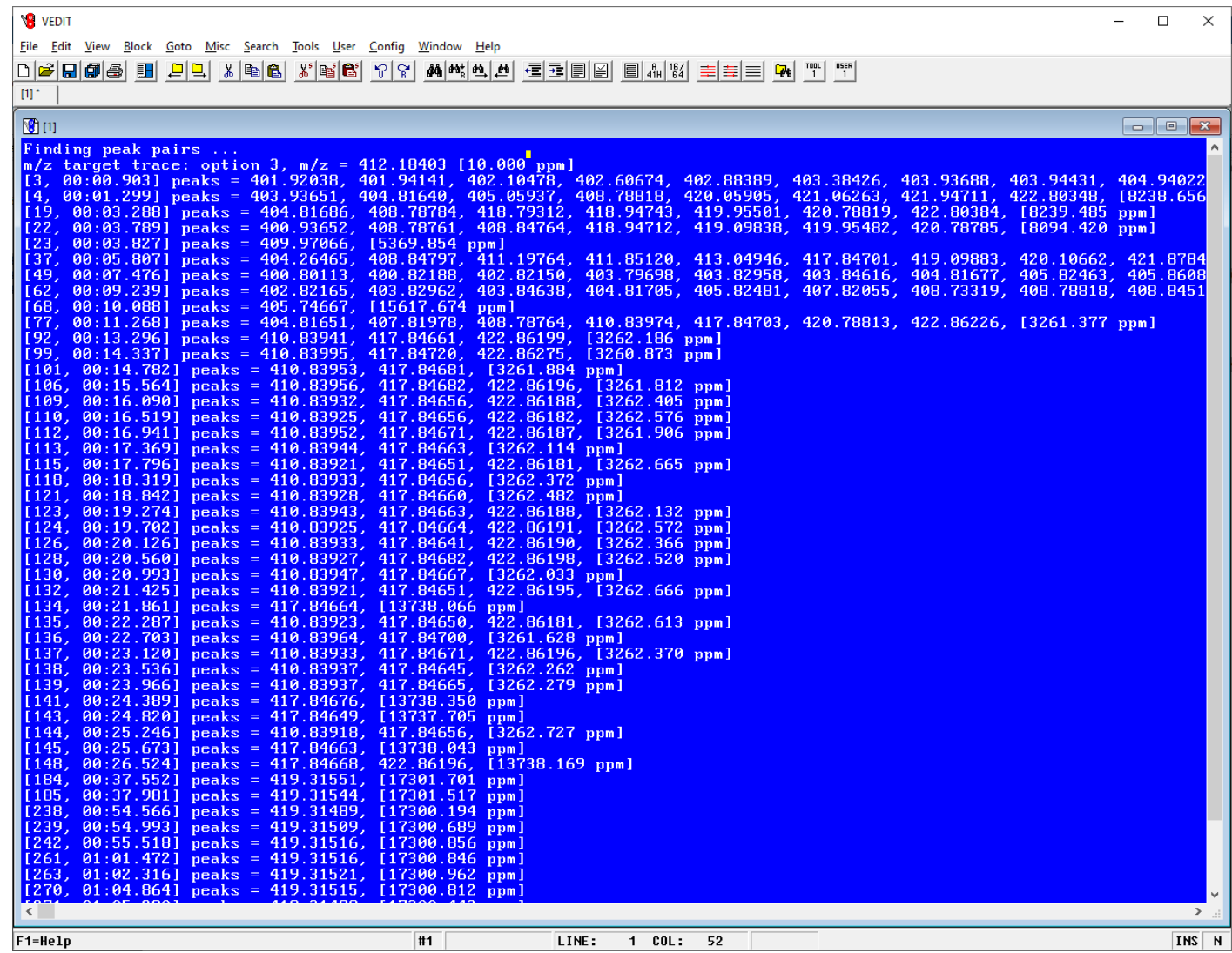

Figure 17. Target peak finder tracing command window output. 

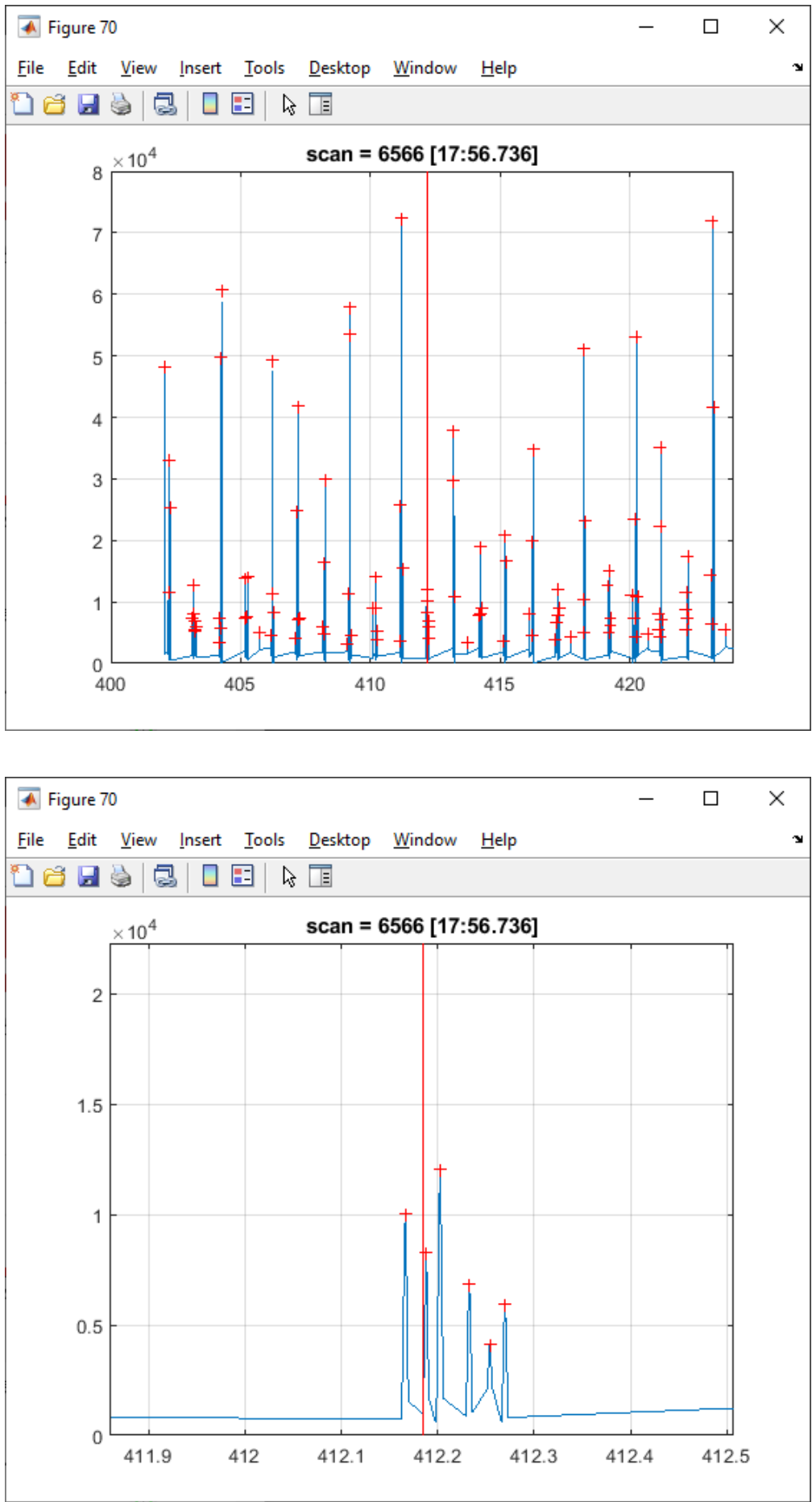

Figure 18. Target peak finder tracing scan plot: the $\mathrm{m} / \mathrm{z} \mathrm{ROI}$ (upper) and the peaks closest to the target $\mathrm{m} / \mathrm{z}$ (lower). 


\subsection{Saving results with Export Peaks}

Immediately after search results are available, the Export Peaks button on the GlycoHunter user interface is enabled. Clicking it launches the Export Peaks user interface shown in Figure 19. Two types of abundance plot are available for visualizing the search results and all search results can be exported to one or more Excel workbooks. For a very large number of peak pairs the Excel limitations on the maximum number of rows or columns may be exceeded. The abundance matrix plots may also be disabled if sufficient memory is not available to build the abundance matrix. A region of interest can be defined to limit the amount of data plotted or saved and the results can be saved or plotted individually for each charge state. Initially, the $\mathrm{ROI}$ is set to the full range of the peak pairs that were found by the search. The right click context menu on the four ROI edit boxes can be used to reset the ROI to these initial values.

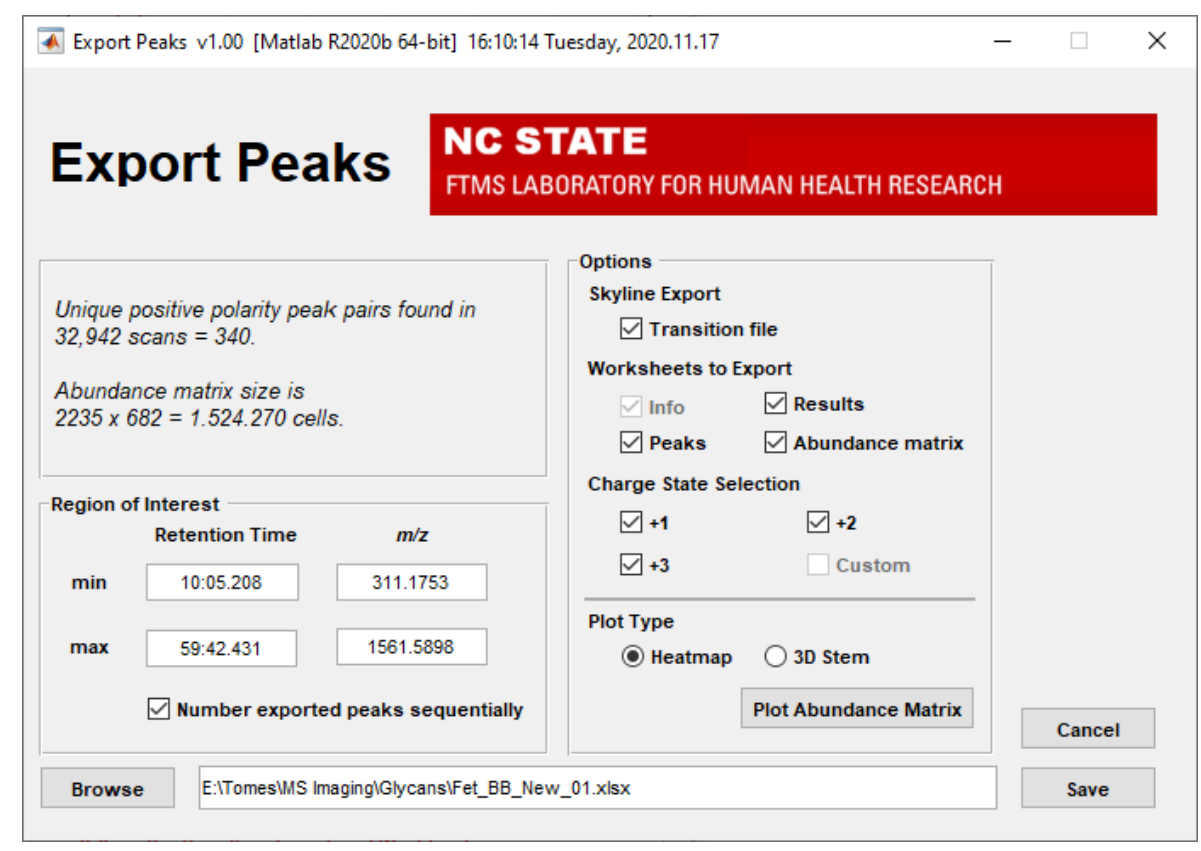

Figure 19. The Export Peaks user interface.

To prevent the user's computer from locking up when building or plotting a very large abundance matrix a percentage of available memory that will be used for the abundance matrix can be specified. This is done with the MaxMemoryPercentage preferences INI file variable. The default value is $68 \%$ of available memory. There are also INI file variables to restrict the size of 
an Excel worksheet. The default values for MaxExcelCols and MaxExcelRows are the Excel limits of 16384 and 1048576, respectively. See Section 3.

Figure 20 shows examples of the two abundance matrix plots: a retention time vs $\mathrm{m} / \mathrm{z}$ heatmap where color is used to represent abundance and a 3D stem plot of retention time vs $\mathrm{m} / \mathrm{z}$ where peak height is proportional to abundance.
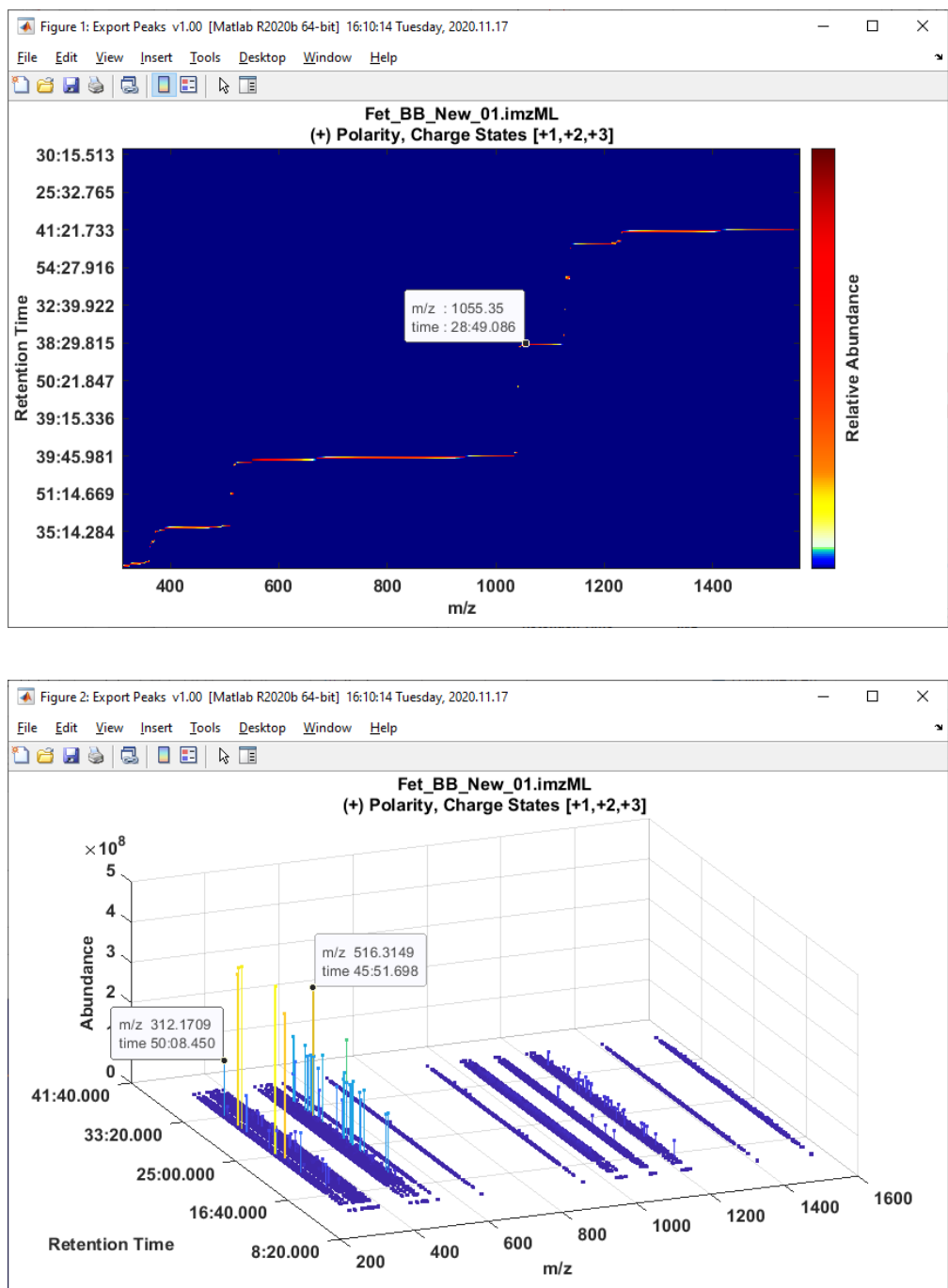

Figure 20. Abundance matrix plots: heatmap (upper), 3D stem plot (lower). In both plots the Matlab data cursor tool has been customized to display the $\mathrm{m} / \mathrm{z}$ and retention time values for any queried data point. 
Depending on the options selected with the Export Peaks GUI, up to six worksheets can be generated in a saved workbook. They are described below.

Info: Contains information about the version of GlycoHunter used to export the data, the name of the data set, number of scans, filter options, region of interest, charge state selection and $\mathrm{m} / \mathrm{z}$ offsets, search parameters, and a summary of the results. The data in this worksheet contains all of the information needed to replicate the search for the same or another data set. An example is shown in Figure 21.

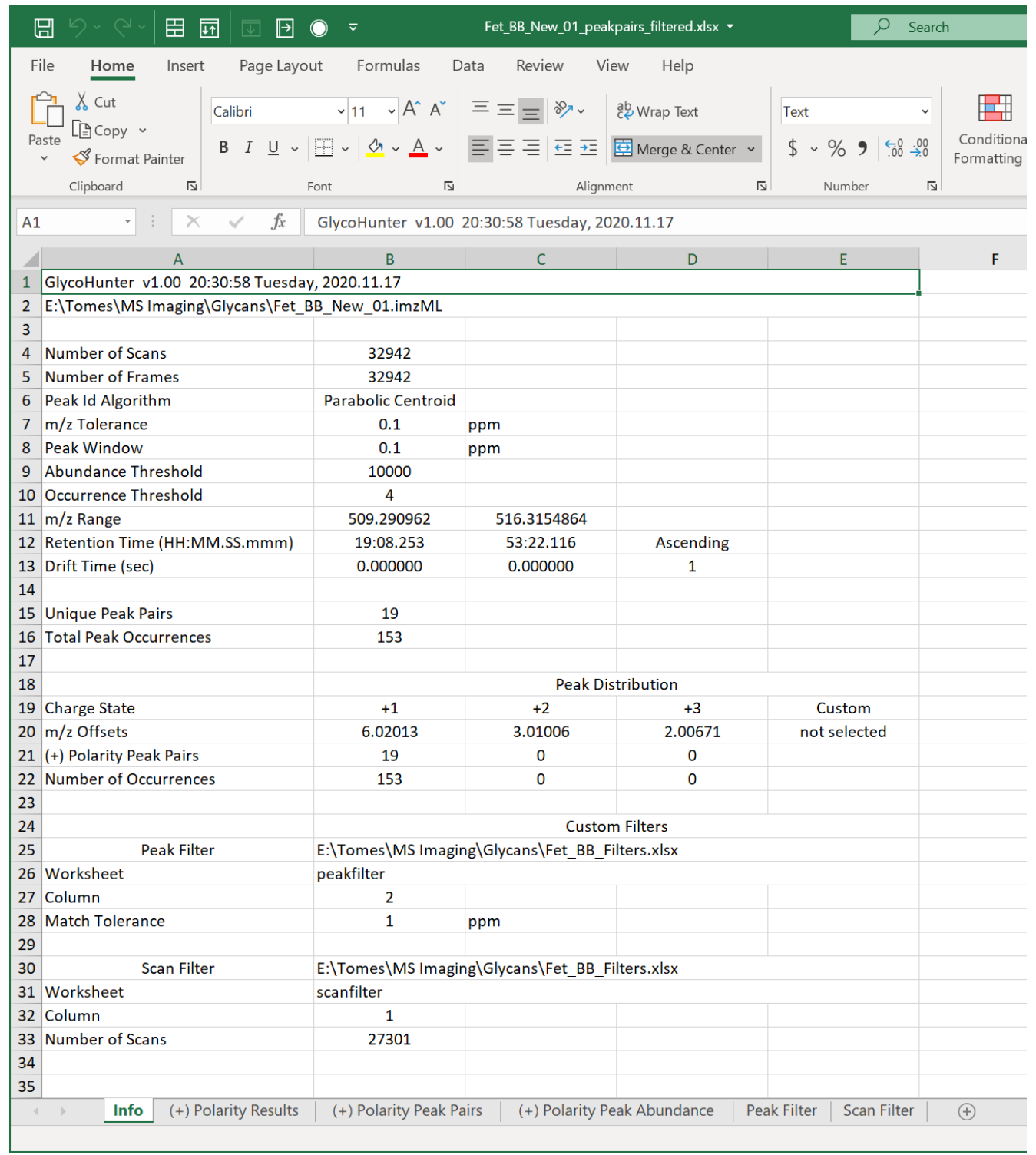

Figure 21. Info worksheet. 
Polarity Results: A list of all peak pairs found that match the search criteria sorted in ascending order by the $\mathrm{m} / \mathrm{z}$ low value (i.e., the monoisotopic peak in each pair). For each pair, the abundance ratio (high $\mathrm{m} / \mathrm{z}$ divided by low $\mathrm{m} / \mathrm{z}$ ), normalized abundance ratio, and the number of occurrences for each charge state are also included. An example is shown in Figure 22. The normalized abundance ratio for each peak occurrence is the sum of the high to low ratio values for that peak (Column K) times the ratio of sum of the low values (Column I) to the sum of the high values (Column J).

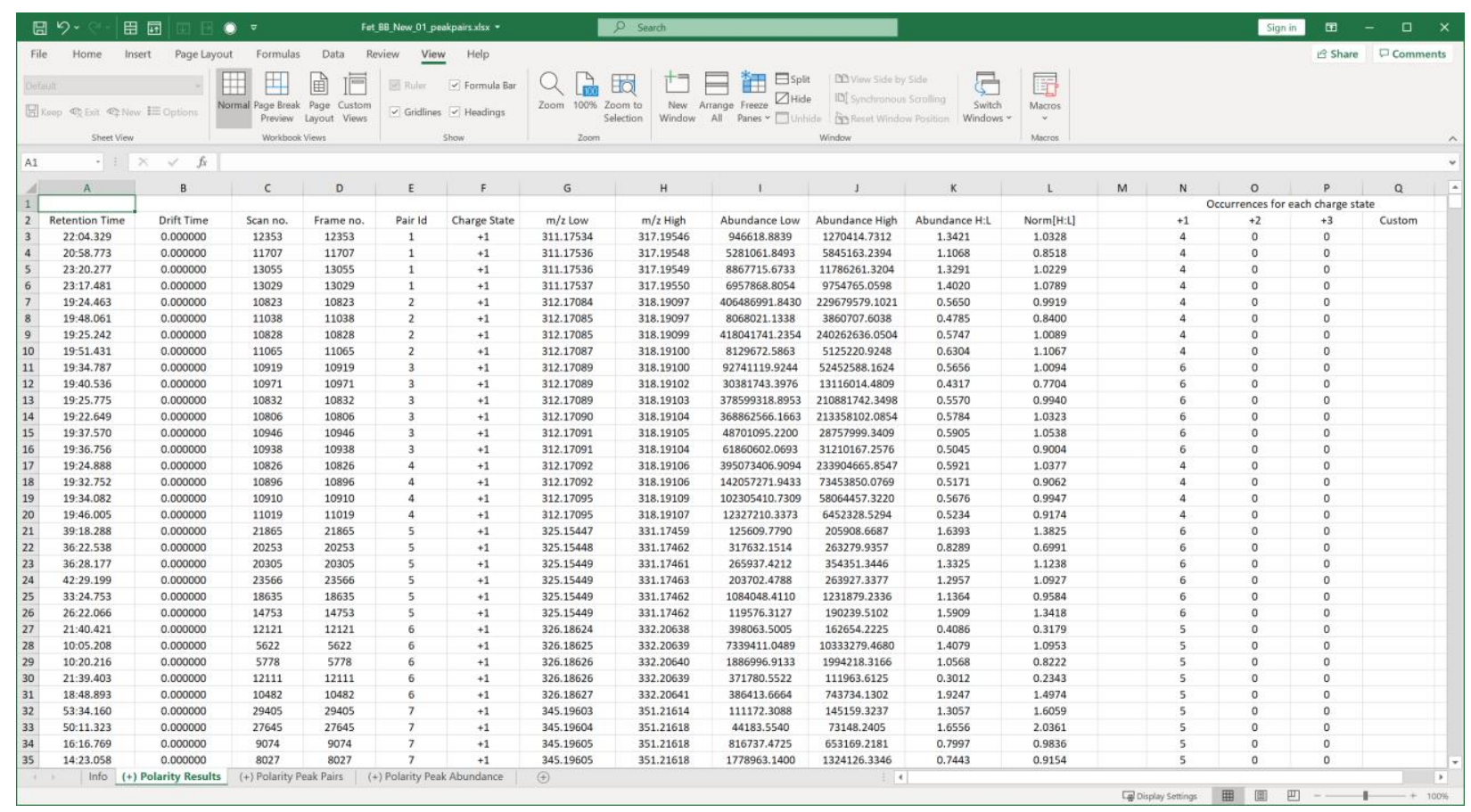

Figure 22. Search results worksheet.

If an ion mobility data set is loaded (imzML format only), columns $B$ and $D$ will contain the drift time and the frame number of each peak pair. Multiple occurrences of a peak pair with different drift times may be separated into potentially isomeric groups by sorting by either of these columns. This is the only support for ion mobility data in this version of GlycoHunter. 
Polarity Peak Pairs: The peak pairs after combining multiple occurrences and grouping by charge state. A portion of an example is shown in Figure 23. There are ten columns of information for each charge state: an id number for the peak pair, the $m / z$ low and high values, the number of occurrences, the mean of the abundance ratios for the peak pair, the mean of the normalized ratios, the standard deviation of the normalized ratios, and the average ratio (Column F divided by Column E).

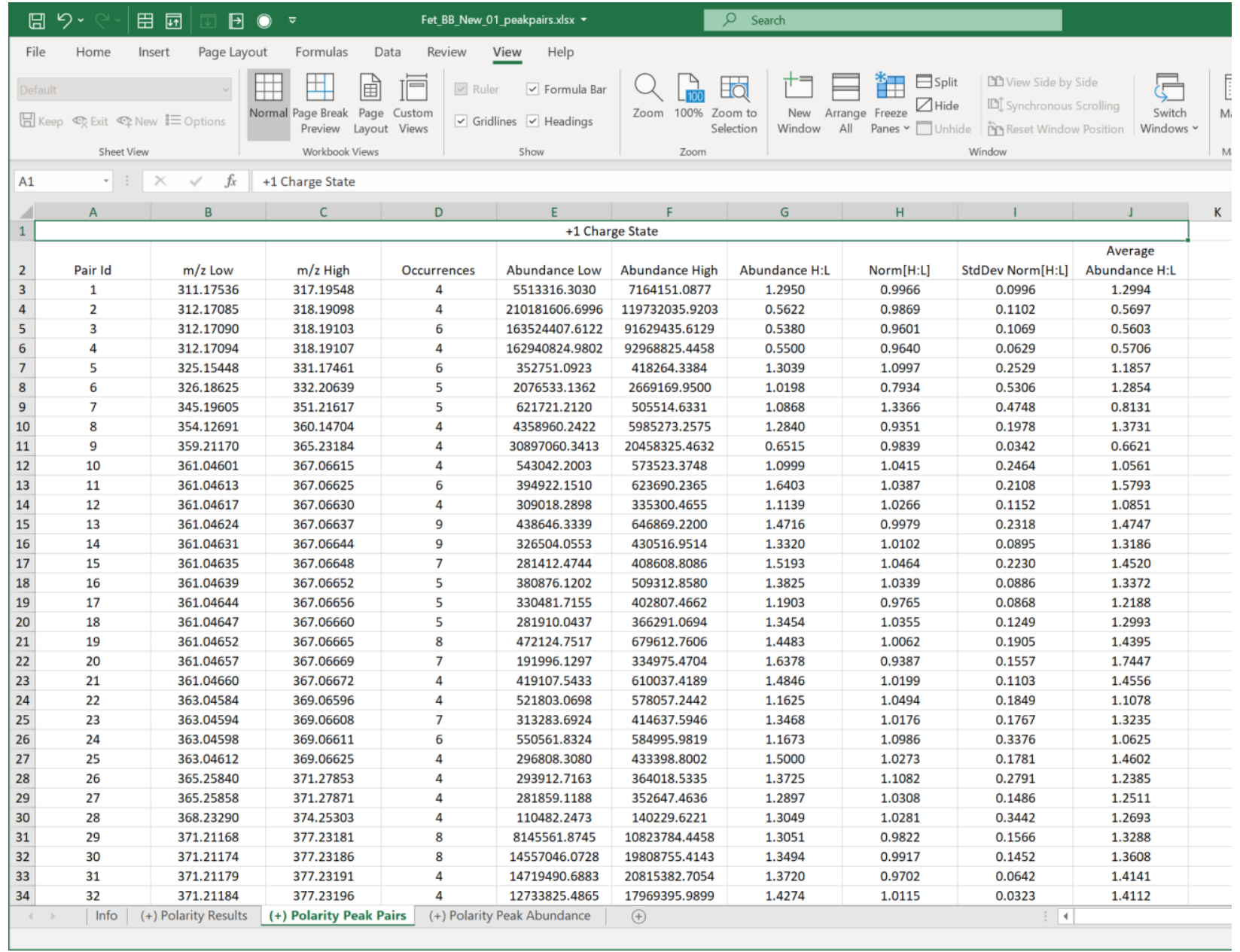

Figure 23. Peak pair results worksheet. 
Polarity Peak Abundance: The data in the Polarity Results worksheet is rearranged into a sparse abundance matrix as shown in Figure 24.

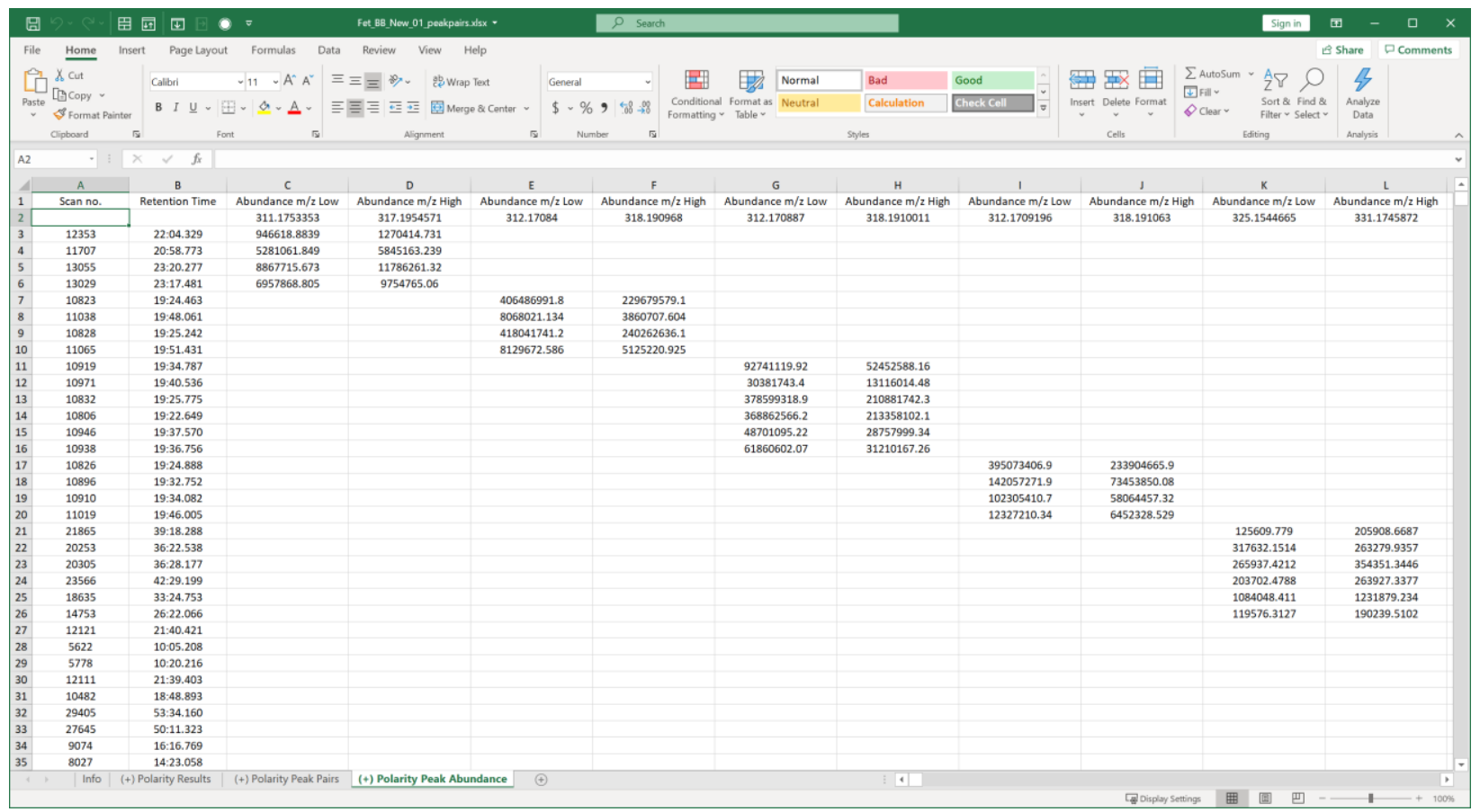

Figure 24. Abundance matrix worksheet. 
Peak Filter: The peak filter worksheet is automatically included if a peak filter was enabled. It is simply a list of the target $\mathrm{m} / \mathrm{z}$ values as shown in Figure 25 (left). The match tolerance is given in the Info worksheet.

Scan Filter: If a scan filter was enabled, the scan numbers are saved in this worksheet. See Figure 25 (right).

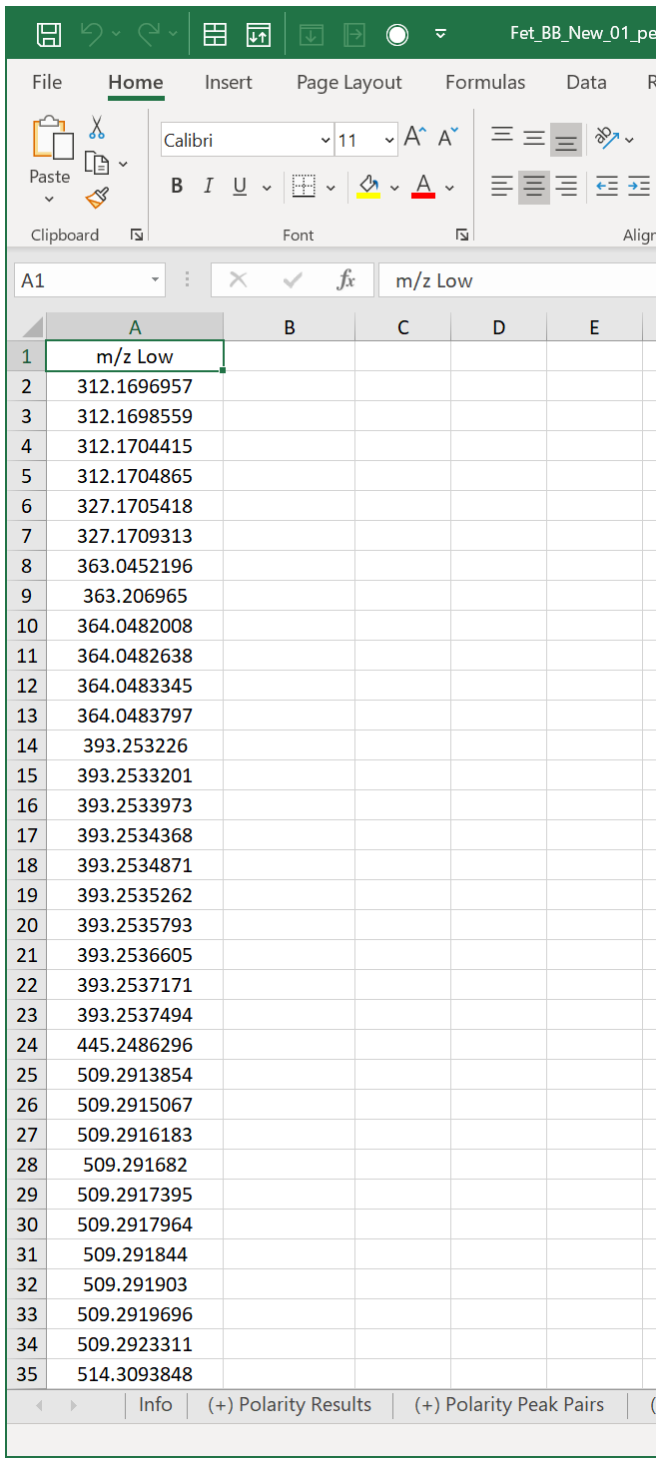

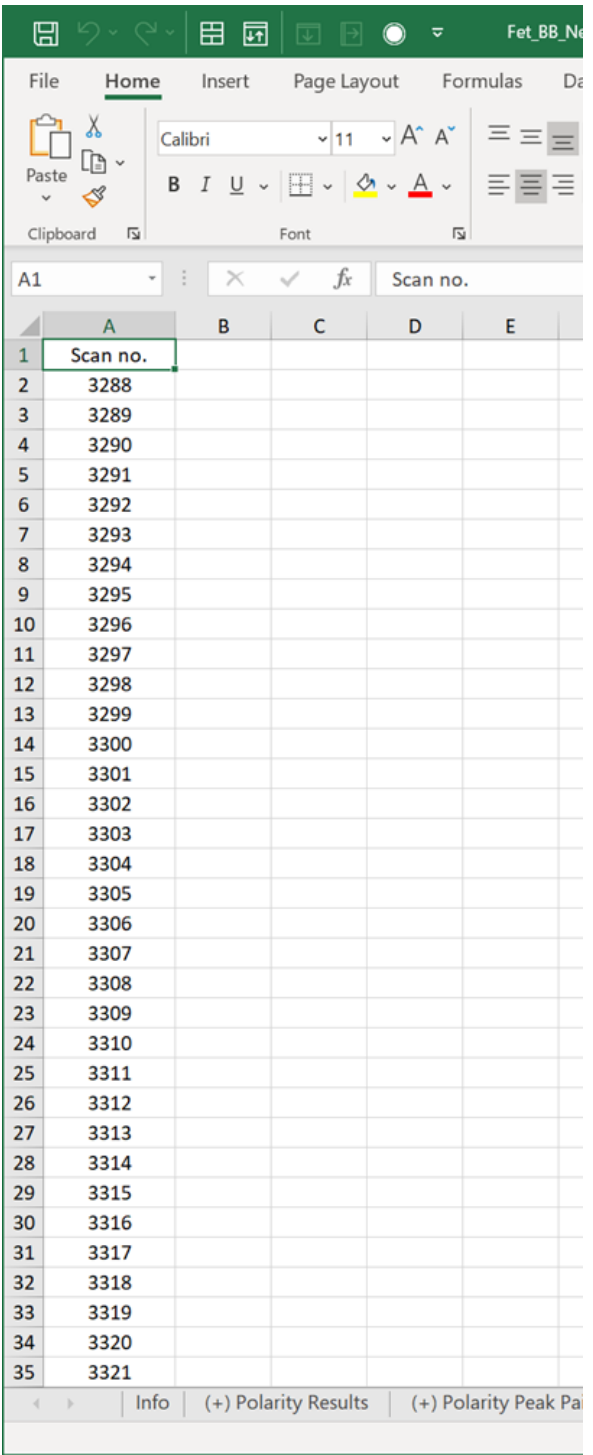

Figure 25. Peak filter (left) and scan filter (right) worksheets. 


\subsection{Skyline transition lists}

The peak pairs can also be saved into an Excel CSV file in a format appropriate for import into Skyline [13,14] as a transition list. Skyline is free and can be downloaded from its website [15]. The website contains in-depth tutorials, webinars, and user group meeting presentation. A stepby-step example is given below using the Skyline-daily tool.

An example of a transition file is shown in Figure 26. The precursor labels "light" and "heavy" can be changed in the preferences INI file (See Section 3).

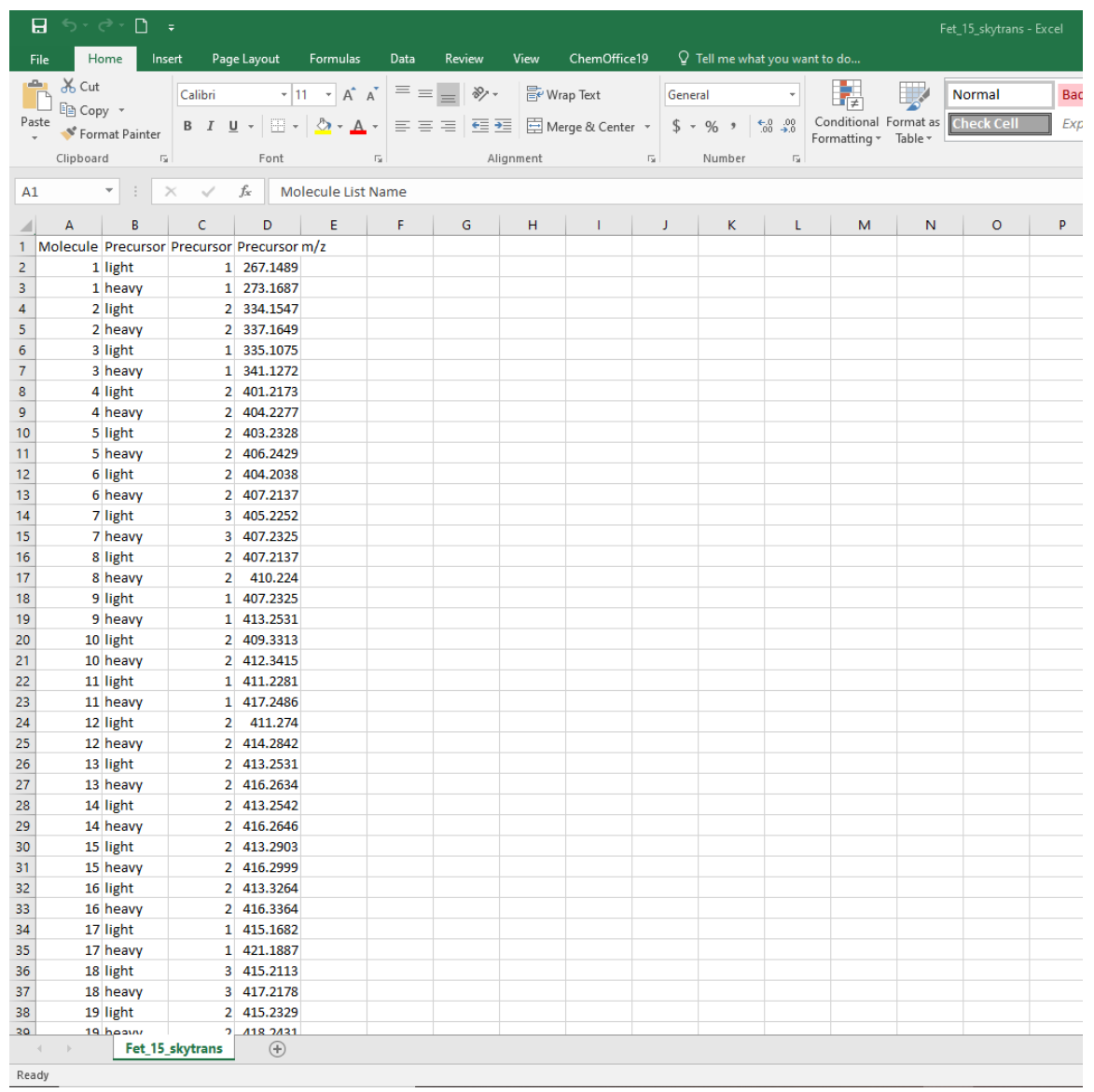

Figure 26. The peak pair search results exported to a Skyline transition list.

The Skyline start page is shown in Figure 27. From this page open a new blank document. The Skyline workspace will be empty and there will be an empty target list (Figure 28). From the Instrument tab of the dialog box click Settings $\rightarrow$ Transition Settings and enter the $\mathrm{m} / \mathrm{z}$ range of the data in your transition list file. 


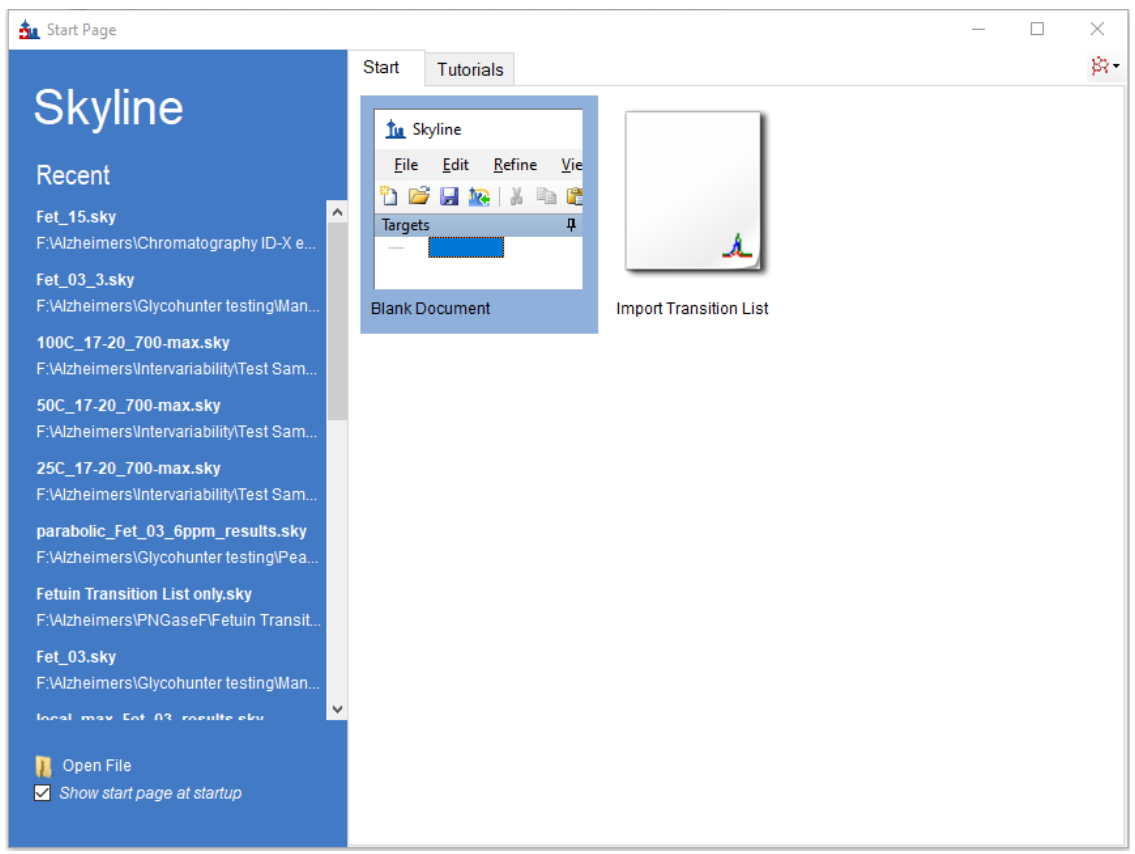

Figure 27. The Skyline start page.

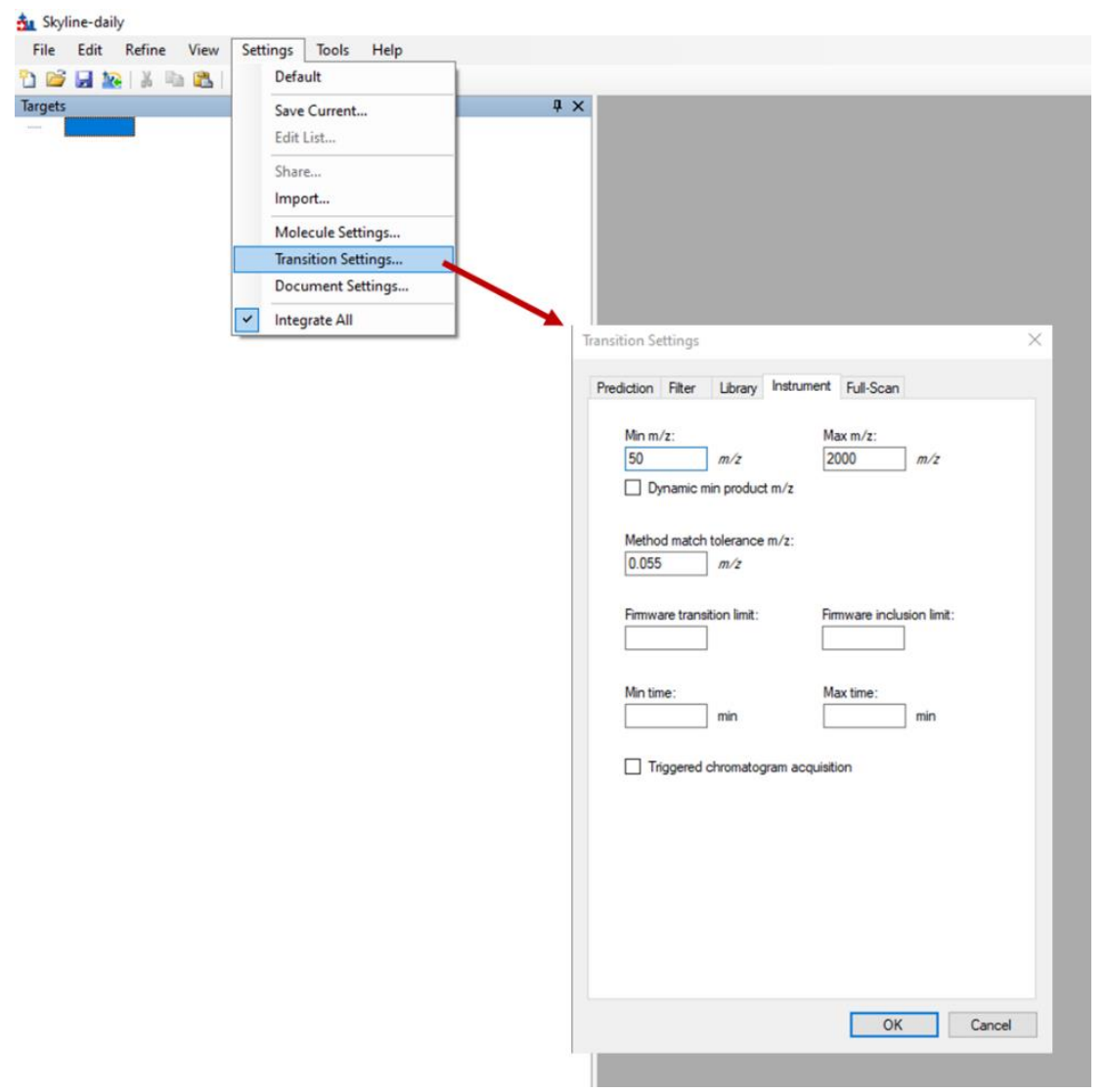

Figure 28. Defining transition list settings in a new Skyline document. 
To load a GlycoHunter transition list use the Skyline File $\rightarrow$ Import $\rightarrow$ Transition List menu to select files saved with Export Peaks as shown in Figure 29. By default, GlycoHunter saves files in the same place as the loaded data set using the same name. Transition list files have the suffix "_skytrans" appended. This can be changed in the preference INI file (Section 3). If multiple files are chosen and the Skyline "add single- injection replicate in files" option has been selected each file will be loaded into a separate chromatographic tab.

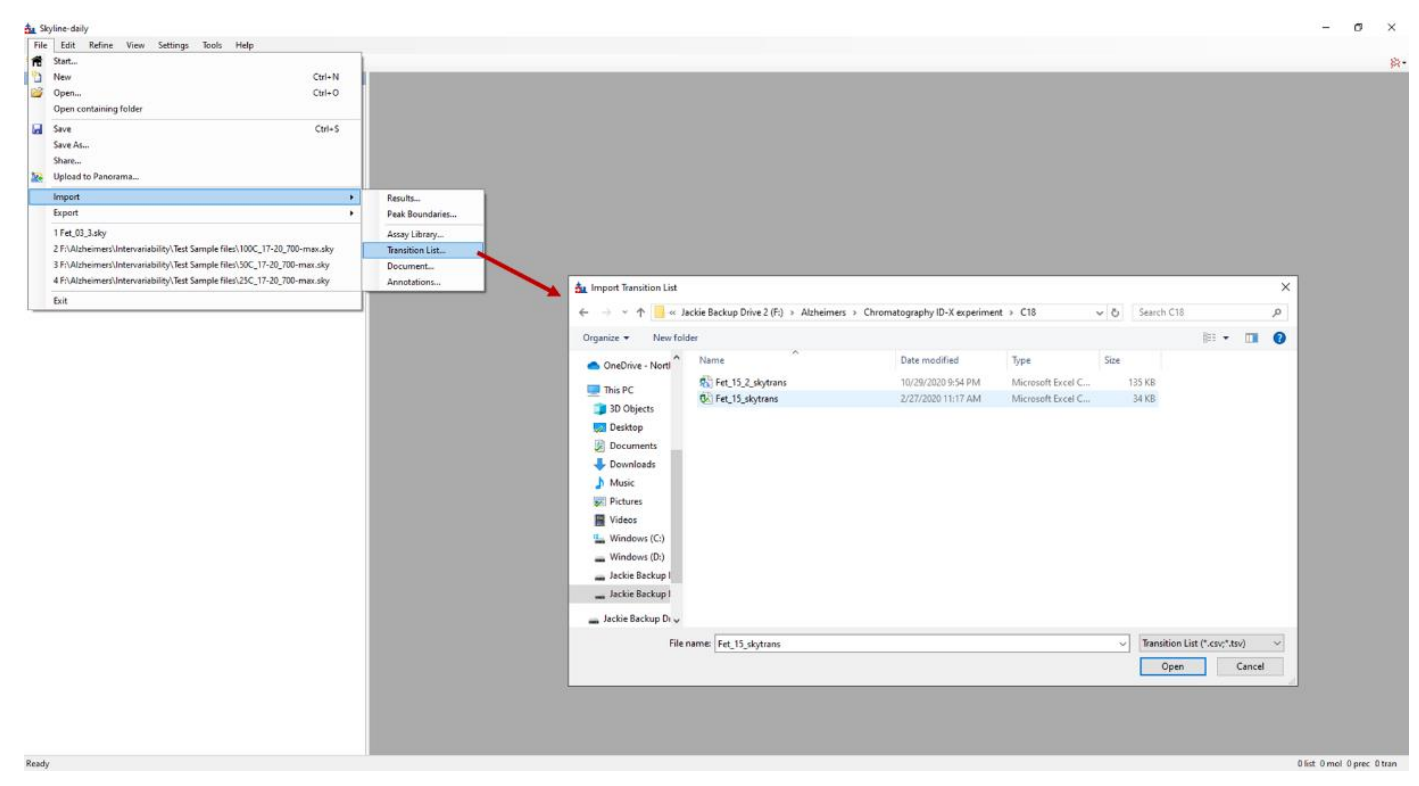

Figure 29. Importing a GlycoHunter transition list into Skyline.
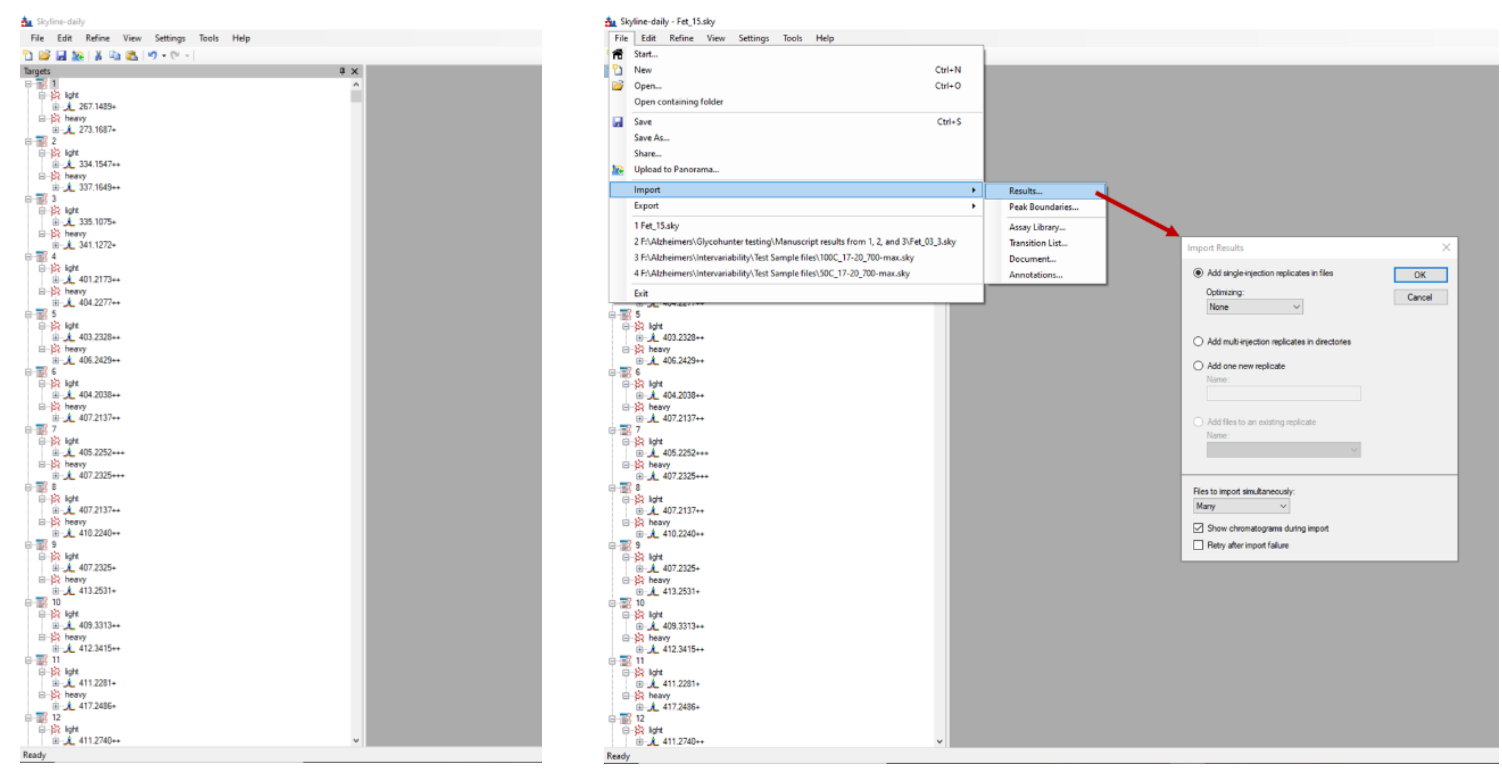

Figure 30. Skyline data analysis: the imported list of target peaks (left), selecting results (right). 
To add additional data analysis tools use the View menu to select analysis windows. In Figure 31 the replicate comparison options of retention time, peak area, and mass errors are shown for a single peak pair. Each $\mathrm{m} / \mathrm{z}$ of the pair can be analyzed separately by clicking on its value in the Targets list. To display the full spectrum, click the top of the peak in the chromatogram window (dot highlighted in red). Mass error values can only be viewed for one $\mathrm{m} / \mathrm{z}$ at a time. The peak area integrations can be manually changed in the chromatogram tab by left clicking under the retention axis at the beginning of the peak and holding the mouse as the dotted lines move to either side of the full peak.

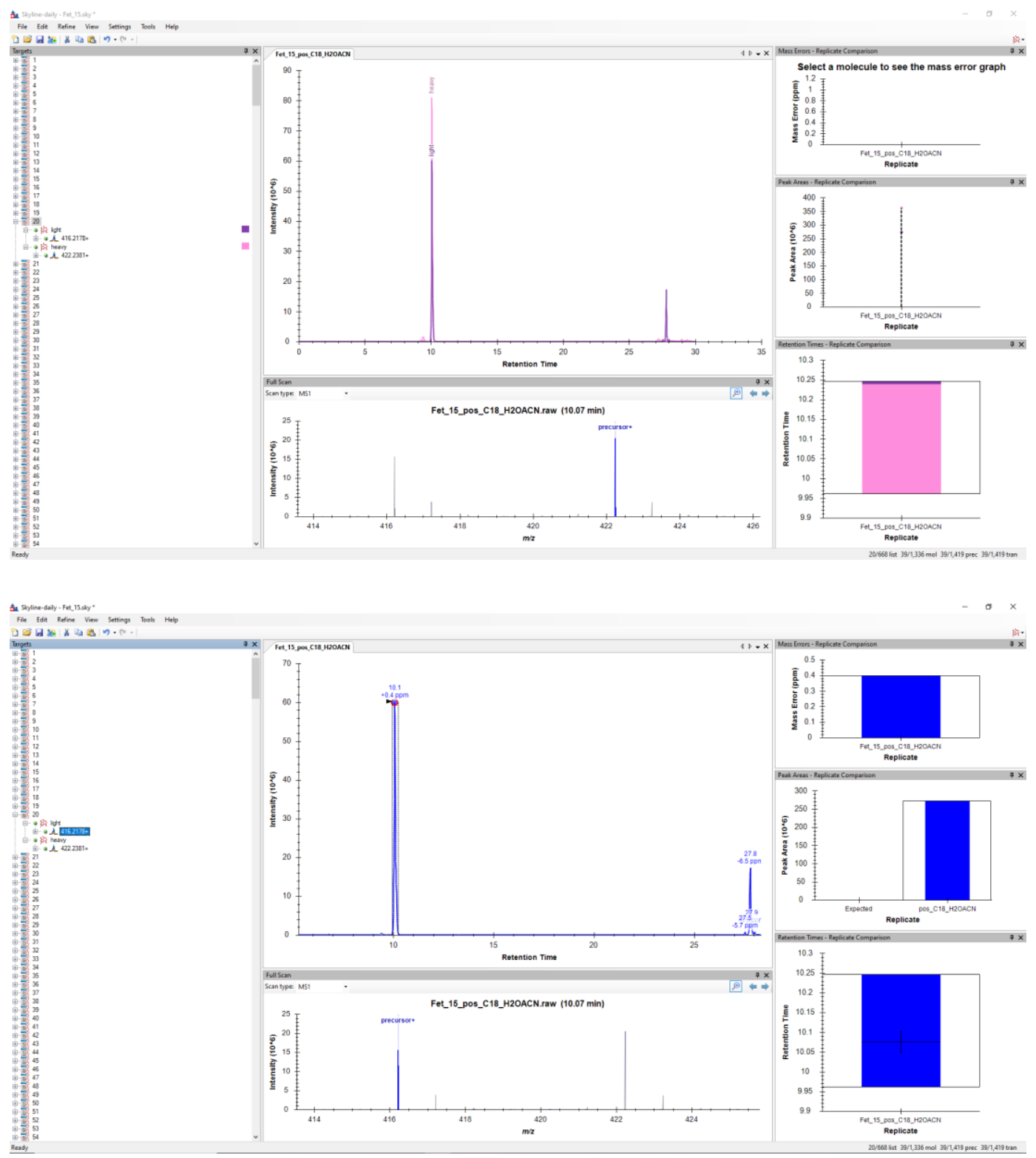

Figure 31. Skyline analysis of the peak pair [415.2178, 422.2381] m/z. 
A "good" putative identification is found when the light and heavy retention times match, the MMA of each peak is below 3 ppm and the light and heavy $\mathrm{m} / \mathrm{z}$ values corresponds to the monoisotopic peak in the full scan window. If the peak pair does not match these criteria, the entry can be removed from the target list on the right by clicking on its ID number and pressing the delete key. 


\section{Customizing GlycoHunter - the preferences INI file}

A plain text preferences file is read by GlycoHunter on startup that contains default values for all settings, options, and search parameters. Editing it allows the user to easily change settings without having to modify source code and to custom GlycoHunter so the default edit box values and checkbox options are the ones that you commonly use. The file is stored in the GlycoHunter installation folder. It is named GlycoHunterPrefs. INI and contains the fields in the table below in the same order that they are listed in the table. 


\begin{tabular}{|c|c|c|}
\hline Parameter Name & Default & Description \\
\hline \multicolumn{3}{|l|}{ [GlycoHunterPrefs] } \\
\hline \multicolumn{3}{|l|}{ GlycoHunter GUI } \\
\hline ChargeStateLabels & $\begin{array}{l}\text { ‘+1’ } \\
‘+2 ’ \\
‘+3 ’ \\
\text { ‘Custom' }\end{array}$ & $\begin{array}{l}\text { A vector of four } m / z \text { offset labels. They should be entered on the same line, } \\
\text { separated by spaces, and enclosed in single quotes. }\end{array}$ \\
\hline ChargeStateTitles & $\begin{array}{l}\text { 'Charge State' } \\
\text { 'Charge States' }\end{array}$ & $\begin{array}{l}\text { Singular and plural forms of the } \mathrm{m} / \mathrm{z} \text { offset labels. They should be entered } \\
\text { on the same line, separated by a space, and enclosed in single quotes. }\end{array}$ \\
\hline ChargeStateValues & $\begin{array}{l}6.20129027 \\
3.010064513 \\
2.006709676 \\
1.505025\end{array}$ & Default $m / z$ offset values. The values should be entered on the same line. \\
\hline ChargeStateMask & true true true false & Default state of the four $\mathrm{m} / \mathrm{z}$ offset checkboxes. \\
\hline mzPrecision & 5 & Precision of displayed $\mathrm{m} / \mathrm{z}$ values. \\
\hline mzTolerance & 0.1 & Default tolerance for matching $\mathrm{m} / \mathrm{z}$ values. \\
\hline mzToleranceUnits & ppm & Default units of $m z$ Tolerance. Allowed values are $p p m$ and $m / z$. \\
\hline PeakWindow & 0.1 & Default peak window width. \\
\hline PeakWindowUnits & ppm & Default peak window units. Allowed values are $p p m$ and $m / z$. \\
\hline PeakldAlgorithm & Parabolic Centroid & $\begin{array}{l}\text { Default peak identification algorithm. Allowed values are Parabolic } \\
\text { Centroid, MS Peaks, and Local Maxima. }\end{array}$ \\
\hline
\end{tabular}




\begin{tabular}{|c|c|c|}
\hline AbundanceThreshold & 10000 & Default value of the abundance threshold for peak identification. \\
\hline OccurrenceThreshold & 4 & $\begin{array}{l}\text { Default value for the minimum number of occurrences for a peak to be } \\
\text { included in the results. }\end{array}$ \\
\hline MinAbundanceThreshold & 0.001 & $\begin{array}{l}\text { Abundance threshold for a data point to be saved when loading a file. } \\
\text { Smaller values are removed from each scan. }\end{array}$ \\
\hline mzFilterMin & 0 & Minimum $m / z$ value allowed when filtering input scan data. \\
\hline mzFilterMax & Inf & Maximum $\mathrm{m} / \mathrm{z}$ value allowed when filtering input scan data. \\
\hline PeakPairSearch & ascending & $\begin{array}{l}\text { Peak pair search direction. The allowed values are ascending and } \\
\text { descending. }\end{array}$ \\
\hline MultiPeakMatchWarning & false & $\begin{array}{l}\text { Set to true to enable message display to the command window whenever } \\
\text { multiple matches for a charge state are found. Multiple matches are always } \\
\text { acknowledged in the peak finder summary dialog. }\end{array}$ \\
\hline PeakFilterTolerance & 1 & Tolerance value for the custom peaks filter. Units are $p p m$. \\
\hline PeakFinderTrace & false & $\begin{array}{l}\text { Set to true to enable verbose message logging to the command window } \\
\text { while searching for peak pairs. }\end{array}$ \\
\hline TICPlotAfterLoad & true & Set to false to disable the TIC plot in the GlycoHunter user interface. \\
\hline PeakFilterHotSheet & Centroid Data & Default name for selecting a peak filter worksheet. \\
\hline ScanFilterHotSheet & Abundance Matrix & Default name for selecting a scan filter worksheet. \\
\hline Pathname & \multicolumn{2}{|c|}{$\begin{array}{l}\text { Default starting path when selecting a file. Do not enclose the path in quotes. } \\
\text { e.g. C:IUsers }|K e n| D o c u m e n t s|M S D a t a|\end{array}$} \\
\hline \multicolumn{3}{|l|}{ Export Peaks GUI } \\
\hline SequentialPeaks & true & Default state of the Number exported peaks sequentially checkbox. \\
\hline
\end{tabular}




\begin{tabular}{|c|c|c|}
\hline SkylineExport & true & Default state of the Skyline Export checkbox. \\
\hline ResultsSheet & true & Default state of the Results checkbox. \\
\hline PeaksSheet & true & Default state of the Peaks checkbox. \\
\hline AbundanceSheet & true & Default state of the Abundance matrix checkbox. \\
\hline PlotType & Heatmap & Default type of plot. The allowed values are Heatmap and 3D Stem. \\
\hline ExportToMAT & false & $\begin{array}{l}\text { Set to true to enable saving search results into a Matlab .MAT format file } \\
\text { when exporting data. }\end{array}$ \\
\hline \multicolumn{3}{|l|}{ Peak Search Tracing } \\
\hline mzTargetTrace & 0 & $\begin{array}{l}\text { Set to a non-zero value to enable a detailed trace of the search process for } \\
\text { a single peak. Allowed values are, } \\
\text { 0, disable } \mathrm{m} / \mathrm{z} \text { target tracing } \\
\text { 1, print messages to the command window } \\
\text { 2, print messages and plot scans in realtime (i.e., in one dynamic plot). } \\
\text { 3, print messages and plot results for each scan with a "hit" } \\
\text { 4, print messages and plot results for each scan individually (i.e., each } \\
\text { result is plotted in a new figure window). }\end{array}$ \\
\hline mzTarget & 412.184027 & Target $m / z$ value for the peak tracing feature \\
\hline mzTargetTolerance & 10 & Tolerance window for mzTarget. Units are ppm. \\
\hline \multicolumn{3}{|l|}{ Memory } \\
\hline ResultMatrixMemAlloc & $1 \mathrm{e} 7$ & $\begin{array}{l}\text { Initial memory allocation for raw peak pairs search results. This is not a } \\
\text { limitation on the maximum number of pairs that can be found. The storage } \\
\text { structure will automatically be increased up to the limit of available RAM; }\end{array}$ \\
\hline
\end{tabular}




\begin{tabular}{|c|c|c|}
\hline & & however, performance is significantly reduced when re-allocation occurs. \\
\hline MaxMemoryPercentage & 0.68 & $\begin{array}{l}\text { Percentage of available memory that can be consumed by Export Peaks } \\
\text { when creating an abundance matrix. }\end{array}$ \\
\hline AbundancePlotSizeWarning & $5 e 6$ & $\begin{array}{l}\text { Threshold number of elements in an abundance matrix at which user } \\
\text { confirmation is required prior to rendering a heatmap or 3D stem plot. }\end{array}$ \\
\hline \multicolumn{3}{|l|}{ Skyline } \\
\hline SkylineFileSuffix & _skytrans & A character string used to build the name of Skyline transition list files. \\
\hline PrecursorLabels & 'light' 'heavy' & $\begin{array}{l}\text { Vector of two labels for Skyline transition CSV files. They should be entered } \\
\text { on the same line, separated by spaces, and enclosed in single quotes. }\end{array}$ \\
\hline \multicolumn{3}{|l|}{ Excel } \\
\hline MaxExcelCols & 16384 & $\begin{array}{l}\text { Maximum number of columns in an Excel worksheet. This value can be } \\
\text { decreased to limit the size of an exported .XLSX file, but it cannot be } \\
\text { increased. }\end{array}$ \\
\hline MaxExcelRows & 1048576 & $\begin{array}{l}\text { Maximum number of rows in an Excel worksheet. This value can be } \\
\text { decreased to limit the size of an exported .XLSX file, but it cannot be } \\
\text { increased. }\end{array}$ \\
\hline XLSTemplateFilename & EmptyTemplate.xlsx & $\begin{array}{l}\text { Results template file. It must be stored in the GlycoHunter installation } \\
\text { folder. }\end{array}$ \\
\hline
\end{tabular}




\section{Troubleshooting}

\subsection{I get a "Java heap size" error message in the command window}

The default value for Java Heap memory in Matlab is $128 \mathrm{Mb}$. This does not leave enough space to load and process a large data set. The heap space allocation can be changed with the Preference/General/Java Heap Memory setting which is on the home tab for newer Matlab releases and under the File menu for older releases. Increase the heap memory to $500 \mathrm{Mb}$ or $1000 \mathrm{Mb}$ to solve this problem. The maximum heap size allowed depends on the amount of RAM installed. You will have to restart Matlab for the change to take effect.

For the standalone version, the Java Heap memory can also be specified. The file java.opts in the installation directory contains a single line of text, "-Xmx512m". This allocates $512 \mathrm{Mb}$ when the standalone version of GlycoHunter is run. Try increasing the Java memory allocation if you have problems. 


\subsection{Tips for processing large data sets}

1. Remove low abundance data points while loading scans by increasing the input threshold filter parameter. The default value of MinAbundanceThreshold in the INI preferences file is 0.001 .

2. Load only a portion of the $m / z$ range for each scan with the mzFilterMin and mzFilterMax INI preferences file parameters. The default values are zero and Inf.

3. Target your search with the scan and peak filters.

4. Narrow the search range with the retention time and $m / z$ region of interest settings.

5. Search and export separately for each charge state.

6. Convert the file to imzML format instead of using mzXML. The file will load faster and peak memory usage will be lower.

7. Set the Matlab array size limit preference to something less than $90 \%$. This will cause an error message to be generated instead of your crashing or "locking-up" Matlab if GlycoHunter or Export Peaks tries to allocate more memory than the amount of RAM in your computer. Even with virtual memory enabled, when a single application uses more than the physical RAM performance usually becomes unacceptable. 


\section{References}

1. Open Source Initiative OSI - The BSD 3-Clause License. Available from: http://opensource.org/licenses/BSD-3-Clause

2. Römpp, A.S., T.; Hester, A.; Klinkert, I.; Heeren, R.; Stöckli, M.; et al., Data Mining in Proteomics, ed. M.S. Hamacher, C.; Eisenacher, M.;2010, New York: Humana Press.

3. Schramm, T., et al., imzML - A common data format for the flexible exchange and processing of mass spectrometry imaging data. Journal of proteomics, 2012. 75(16): $p$. 5106-10.

4. ProteoWizard. Available from: http://proteowizard.sourceforge.net/.

5. $m z X M L$ converter. Available from:

http://tools.proteomecenter.org/wiki/index.php?title=Formats:mzXML.

6. Race, A.M., I.B. Styles, and J. Bunch, Inclusive sharing of mass spectrometry imaging data requires a converter for all. Journal of proteomics, 2012. 75(16): p. 5111-2.

7. Race, A. and A. Römpp, Error-free data visualisation and processing through imzML and mzML validation. Analytical Chemistry, September 17, 2018.

https://pubs.acs.org/doi/10.1021/acs.analchem.8b03059.

8. imzMLValidator. Available from: https://gitlab.com/imzML/imzMLValidator.git.

9. Pedrioli, P.G., et al., A common open representation of mass spectrometry data and its application to proteomics research. Nature biotechnology, 2004. 22(11): p. 1459-66.

10. Giancaspro, C. and M.B. Comisarow, Exact Interpolation of Fourier-Transform Spectra. Applied Spectroscopy, 1983. 37(2): p. 153-166. 
11. Verdun, F.R., T.L. Ricca, and A.G. Marshall, Beating the Nyquist Limit by Means of Interleaved Alternated Delay Sampling: Extension of Lower Mass Limit in Direct-Mode Fourier Transform Ion Cyclotron Resonance Mass Spectrometry. Applied Spectroscopy, 1988. 42(2): p. 199-203.

12. The Mathworks. http://www.mathworks.com/.

13. Pino, L. K.; Searle, B. C.; Bollinger, J. G.; Nunn, B.; Maclean, B.; Maccoss, M. J. The Skyline Ecosystem: Informatics for Quantitative Mass Spectrometry Proteomics. Mass Spectrom. Rev. 2017, 39 (3), 229-244.

14. MacLean, B. X.; Pratt, B. S.; Egertson, J. D.; MacCoss, M. J.; Smith, R. D.; Baker, E. S. Using Skyline to Analyze Data-Containing Liquid Chromatography, Ion Mobility Spectrometry, and Mass Spectrometry Dimensions. J. Am. Soc. Mass Spectrom. 2018, 29 (11), 2182-2188.

15. Skyline. https://skyline.ms/project/home/software/Skyline/begin.view/ 